\title{
Iterative Signal Processing for Mitigation of Analog-to-Digital Converter Clipping Distortion in Multiband OFDMA Receivers
}

\author{
Markus Allén, Toni Levanen, Jaakko Marttila, and Mikko Valkama \\ Department of Communications Engineering, Tampere University of Technology, P.O. Box 553, 33101 Tampere, Finland \\ Correspondence should be addressed to Markus Allén, markus.allen@tut.fi
}

Received 31 May 2011; Revised 23 October 2011; Accepted 31 October 2011

Academic Editor: Tzi-Dar Chiueh

Copyright () 2012 Markus Allén et al. This is an open access article distributed under the Creative Commons Attribution License, which permits unrestricted use, distribution, and reproduction in any medium, provided the original work is properly cited.

In modern wideband communication receivers, the large input-signal dynamics is a fundamental problem. Unintentional signal clipping occurs, if the receiver front-end with the analog-to-digital interface cannot respond to rapidly varying conditions. This paper discusses digital postprocessing compensation of such unintentional clipping in multiband OFDMA receivers. The proposed method iteratively mitigates the clipping distortion by exploiting the symbol decisions. The performance of the proposed method is illustrated with various computer simulations and also verified by concrete laboratory measurements with commercially available analog-to-digital hardware. It is shown that the clipping compensation algorithm implemented in a turbo decoding OFDM receiver is able to remove almost all the clipping distortion even under significant clipping in fading channel circumstances. That is to say, it is possible to nearly recover the receiver performance to the level, which would be achieved in the equivalent nonclipped situation.

\section{Introduction}

Modern wideband radio receivers, such as cognitive radios, are setting significant challenges for the design of the receiver front-end. One of the key issues is to have enough dynamic range $[1,2]$. Current communication waveforms tend to have a high peak-to-average-power ratio (PAPR), which makes it challenging to optimize the usage of power amplifier on the transmitter side, and it can also increase the required dynamic range in the receiver. Moreover, the reception becomes even more challenging, if a wide frequency band with several independent signals is received with a single receiver front-end and A/D interface. Those independent signals can have considerably different power levels and hence the required dynamic range is not determined only by the PAPR of a single-user signal but by the dynamic range of the overall received waveform $[3,4]$. The power difference between two independent signals in mobile environment can be several tens of $\mathrm{dBs}$, which is a particularly important issue to take into account in cognitive radios. Due to the continuously changing conditions, the automatic gain control (AGC) of the receiver might not be able to follow the signal dynamics. Especially the suddenly appearing strong adjacent-channel signals, with no power control from secondary (cognitive) radio point of view will cause problems. The AGC failure causes too high input level for the A/D converter and therefore the amplitude of the digitized waveform is saturated, that is, the highest signal peaks are clipped. This causes considerable signal distortion especially at the weak signal bands due to intermodulation of strong input components.

This paper discusses the compensation of unintentional receiver clipping occurring in $\mathrm{A} / \mathrm{D}$ converters using digital postprocessing. More specifically, the focus is on orthogonal frequency division multiplexing- (OFDM-) based multiple access scheme, noted in the literature as OFDMA. The reception of a weak signal can be seriously compromised in the presence of a stronger adjacent-channel signal, because clipping causes relatively high amount of nonlinear distortion to the weak signal band of interest. The current literature has only a very limited number of publications proposing digital signal processing methods for reducing unintentional clipping taking place on the receiver side [57]. The aforementioned references contain rather general methods, which can be applied to different kinds of communication systems. Nevertheless, a compensation method, which is designed for a certain system, can perform better from the accuracy or computational complexity point of view. Therefore, this paper specifically focuses on OFDM, 
which is widely used in emerging radio systems, and the receiver clipping compensation methods are likely to be needed. Currently, most of the OFDM-related clipping compensation algorithms in the literature concentrate on deliberate clipping to reduce signal PAPR on the transmitter side [8-10]. These methods are not directly applicable to mitigate clipping occurring on the receiver since the exact clipping level is not known in the case of unintentional clipping. In addition, the receiver-side clipping case is more challenging, because it happens after the radio channel and hence interferes with the channel estimation.

The proposed receiver clipping compensation method relies on the idea that most of the bit decisions made on the receiver are still correct and hence they can be exploited in clipping distortion estimation. This kind of decisionbased digital compensation methods have been proposed in the literature for mitigation of various RF impairments, for example, phase noise [11, 12] and power amplifier nonlinearity [13]. This paper extends the work initiated in [14], which uses the PAPR reduction scheme of $[9,10]$ as a starting point and then modifies it to be suitable for the challenging task of removing unintentional receiverside clipping. This paper provides more thorough signal modeling, performance analysis, and considers the use of turbo codec as well as more advanced channel estimation than what is presented in [14]. Additionally, the performance of the receiver clipping compensation method is verified with laboratory measurements employing commercially available $\mathrm{A} / \mathrm{D}$ hardware. The results show that the proposed receiver clipping compensation method effectively mitigates the clipping distortion, is robust against various estimation errors, and is implementable with reasonable hardware costs in practice.

The remainder of the paper is organized as follows. Section 2 provides background information about the modeling of A/D converter clipping and also defines the notation used in the paper to express $\operatorname{OFDM}(\mathrm{A})$ signals. Section 3 then introduces the postprocessing algorithm for compensating clipping distortion and discusses its implementation details. The performance of the proposed algorithm is carefully studied in Section 4 using computer simulations. In addition, practical laboratory measurements with real A/D hardware are used to verify the functionality of the algorithm. After that, the computational complexity of the algorithm in general is discussed. Section 5 finishes the paper by drawing conclusions.

\section{Received OFDM Signal Model and Clipping Phenomenon}

This paper considers the traditional OFDM signal model, where the transmitted baseband OFDM symbol in time domain is first expressed as

$$
x(t)=\frac{1}{\sqrt{N}} \sum_{k=-N / 2}^{N / 2-1} X_{k} e^{j 2 \pi k t / T_{s}}, \quad 0 \leq t \leq T_{s},
$$

where $X_{k}$ is the $k$ th complex data symbol (subcarrier $k$ ) and $T_{s}$ is the OFDM symbol duration. This OFDM symbol

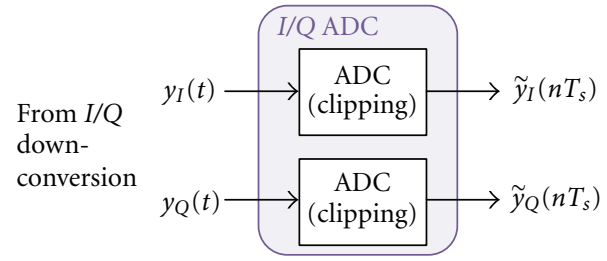

FIGURE 1: Illustration of an $I / Q$ A/D converter, which contains separate converters for the $I$ and $Q$ branches of the received downconverted signal $y(t)=y_{I}(t)+j y_{Q}(t)$.

contains $N_{A}$ active subcarriers (SC) and has an oversampling factor $J$. Therefore, the total amount of SCs is $N=J N_{A}$ and the SC indices are expressed as a set $\Omega=\{-N / 2,-N / 2+$ $1, \ldots, N / 2-1\}$. The active SCs carry the data symbol sequence $X_{k}, k \in \Omega_{A}=\left\{-N_{A} / 2, \ldots,-1,1, \ldots, N_{A} / 2\right\}$, whereas the nonactive SCs contain zeros, that is, $X_{k}=0, k \in$ $\Omega \backslash \Omega_{A}$. It is worth noticing that the presented OFDM signal model does not include cyclic prefix (CP), which is required in practice to avoid intersymbol interference in frequency-selective mobile environments. This paper omits the $\mathrm{CP}$ from the mathematical notation in order to make it simpler. This simplification does not affect the presented clipping analysis in any way, because clipping is a memoryless phenomenon. On the other hand, the CP is taken into account in the proposed receiver structures of Section 3 and in the simulations of Section 4 due to the CP's essential role in implementations of practical communication systems.

A zero-symmetric hard-clipping model is a simple but accurate way to characterize signal clipping in receiver A/D converters. Let us first define a general received waveform as $y(t)=y_{I}(t)+j y_{Q}(t)=h(t) * x(t)+w(t)$, where the noise $w(t)$ is added to the convolution of the channel impulse response $h(t)$ and the transmitted signal $x(t)$. After an $I / Q$ A/D converter (see Figure 1), clipping occurring separately in both branches, the discrete-time received signal is $\tilde{y}\left(n T_{s}\right)=$ $\tilde{y}_{I}\left(n T_{s}\right)+j \tilde{y}_{Q}\left(n T_{s}\right)$, where the zero-symmetric hard-clipping model determines that

$$
\begin{gathered}
\tilde{y}_{I}(t)= \begin{cases}y_{I}(t), & \left|y_{I}(t)\right|<V_{0, I} \\
V_{0, I}, & y_{I}(t) \geq V_{0, I} \\
-V_{0, I}, & y_{I}(t) \leq-V_{0, I},\end{cases} \\
\tilde{y}_{Q}(t)= \begin{cases}y_{Q}(t), & \left|y_{Q}(t)\right|<V_{0, Q} \\
V_{0, Q}, & y_{Q}(t) \geq V_{0, Q} \\
-V_{0, Q}, & y_{Q}(t) \leq-V_{0, Q},\end{cases}
\end{gathered}
$$

where $V_{0, I}$ and $V_{0, Q}$ denote the maximum output levels of the A/D converters of $I$ and $Q$ branches, respectively. Often in practice, a convenient way to express the clipping level of the signal is needed, but $V_{0, I}\left(V_{0, Q}\right)$ by itself is not very descriptive if nothing about the signal is known. Therefore, the common practice is to define the clipping level using a ratio of $V_{0, I}\left(V_{0, Q}\right)$ and the average branch power level of 
the nonclipped signal $P_{\mathrm{in}, I}\left(P_{\mathrm{in}, Q}\right)$. In other words, the clipping level for I branch $\gamma_{I}$ can be written in the form of

$$
\gamma_{I}=\frac{V_{0, I}}{\sqrt{P_{\mathrm{in}, I}}}
$$

and similarly

$$
\gamma_{Q}=\frac{V_{0, Q}}{\sqrt{P_{\text {in, },}}},
$$

for the $Q$ branch. These variables give a precise understanding of how intensely the signal branch is actually clipped. In addition, it can be concluded from (3) and (4) that the clipping level $\gamma$ is different for $I$ and $Q$ branches, if the maximum input levels of the converters are different $\left(V_{0, I} \neq V_{0, Q}\right)$ or the branch signal powers are not equal $\left(P_{\mathrm{in}, I} \neq P_{\mathrm{in}, Q}\right)$. Notice that even though $V_{0, I}$ and $V_{0, Q}$ are most likely designed to be equal, unavoidable implementation tolerances of two physical separate converter circuits are indeed likely to cause small relative deviations between them.

The clipping phenomenon removes the highest signal amplitudes and hence causes nonlinear distortion. This process defined in (2) is not directly invertible. Therefore, another kind of approach is required for compensating the clipping distortion, especially at weak signal bands. This paper exploits Bussgang's theorem [15] for the compensation purpose. It has already been proven to be an adequate way to model the clipping distortion [14, 16-18]. Basically, Bussgang's theorem states that the clipping attenuates the received signal $y\left(n T_{s}\right)$ with a factor $\alpha$ and also causes additive clipping distortion $d\left(n T_{s}\right)$, which is uncorrelated with the nonclipped $y\left(n T_{s}\right)$. Therefore, the clipped signal branches can be expressed as

$$
\begin{aligned}
& \tilde{y}_{I}\left(n T_{s}\right)=\alpha_{I} y_{I}\left(n T_{s}\right)+d_{I}\left(n T_{s}\right), \\
& \tilde{y}_{Q}\left(n T_{s}\right)=\alpha_{Q} y_{Q}\left(n T_{s}\right)+d_{Q}\left(n T_{s}\right),
\end{aligned}
$$

when for modeling purposes $y_{I}\left(n T_{s}\right)$ and $y_{Q}\left(n T_{s}\right)$ are assumed to be Gaussian. Here, $\alpha_{I}$ and $\alpha_{Q}$ depend on $\gamma_{I}$ and $\gamma_{\mathrm{Q}}$, respectively. For the corresponding complex signal $\tilde{y}\left(n T_{s}\right)$, it can be written that

$$
\begin{aligned}
\tilde{y}\left(n T_{s}\right) & =\alpha_{I} y_{I}\left(n T_{s}\right)+d_{I}\left(n T_{s}\right)+j\left[\alpha_{Q} y_{Q}\left(n T_{s}\right)+d_{Q}\left(n T_{s}\right)\right] \\
& =\alpha_{1} y\left(n T_{s}\right)+\alpha_{2} y^{*}\left(n T_{s}\right)+d\left(n T_{s}\right) \\
& =\alpha_{1} y\left(n T_{s}\right)+z\left(n T_{s}\right),
\end{aligned}
$$

where $\alpha_{1}=\left(\alpha_{I}+\alpha_{Q}\right) / 2, \alpha_{2}=\left(\alpha_{I}-\alpha_{Q}\right) / 2$, complex clipping distortion $d\left(n T_{s}\right)=d_{I}\left(n T_{s}\right)+j d_{Q}\left(n T_{s}\right)$, and total interference $z\left(n T_{s}\right)=\alpha_{2} y^{*}\left(n T_{s}\right)+d\left(n T_{s}\right)$. In the general case with unequal clipping levels in the I and $\mathrm{Q}$ branches, the total interference $z\left(n T_{s}\right)$ contains both the nonlinear clipping distortion $d\left(n T_{s}\right)$ and the conjugate signal interference $\alpha_{2} y^{*}\left(n T_{s}\right)$, which in the frequency domain corresponds to mirror-frequency interference. In the special case of identical clipping levels for both branches, $\gamma_{I}=\gamma_{Q}=\gamma$, the attenuation factor is $\alpha=\alpha_{I}=\alpha_{Q}$ and, therefore, $\alpha_{1}=\alpha$ and $\alpha_{2}=0$. This means that the general complex signal model presented in (6) reduces to

$$
\tilde{y}\left(n T_{s}\right)=\alpha y\left(n T_{s}\right)+d\left(n T_{s}\right),
$$

which is in accordance with the traditional Bussgang's theorem for a complex signal.

Even a clipping model as simple as (6) or (7) is justified due to the memoryless nature of the clipping phenomenon. In addition, the model is linear and hence straightforward to utilize in clipping compensation purposes as described in Section 3. In short, if $\alpha_{1}$ and $z\left(n T_{s}\right)$ can be estimated, recovery of the nonclipped signal $y(t)$ is simple using (6). The Gaussianity assumption for $y_{I}\left(n T_{s}\right)$ and $y_{Q}\left(n T_{s}\right)$ also makes it possible to derive concise equations for $\alpha_{I}$ and $\alpha_{Q}$, namely,

$$
\begin{aligned}
& \alpha_{I}=\frac{E\left[y_{I}\left(n T_{s}\right) \tilde{y}_{I}\left(n T_{s}\right)\right]}{E\left[y_{I}\left(n T_{s}\right) y_{I}\left(n T_{s}\right)\right]}=\operatorname{erf}\left(\frac{\gamma_{I}}{\sqrt{2}}\right), \\
& \alpha_{Q}=\frac{E\left[y_{Q}\left(n T_{s}\right) \tilde{y}_{Q}\left(n T_{s}\right)\right]}{E\left[y_{Q}\left(n T_{s}\right) y_{Q}\left(n T_{s}\right)\right]}=\operatorname{erf}\left(\frac{y_{Q}}{\sqrt{2}}\right) .
\end{aligned}
$$

Here the error function $\operatorname{erf}(x)$ is defined as

$$
\operatorname{erf}(x)=\frac{2}{\sqrt{\pi}} \int_{0}^{x} e^{-t^{2}} d t .
$$

The details related to the derivation of (8) are presented in [19].

\section{Iterative Decision-Aided Method for Compensating Unintentional Receiver Clipping}

This section proposes a compensation algorithm for removing signal distortion caused by unintentional clipping in the A/D interface of an OFDM receiver. First, the basic compensation idea and its justification through mathematical analysis are described in Section 3.1 with the help of a block diagram presented in Figure 2. After that, the most essential details from the implementation point of view are discussed in Section 3.2. Unintentional clipping is most likely to happen in multiuser/multiband situations. Therefore, Section 3.3 deals with the multiuser aspects of the compensation algorithm implementation from the uplink (UL) and downlink (DL) point of view.

3.1. The Compensation Algorithm. On top part of Figure 2, a traditional OFDM transmitter exploiting turbo coding is illustrated. After channel coding and interleaving, the coded bits $c_{1, \ldots, M}^{\left(X_{k}\right)}, k \in \Omega_{A}$, are mapped to data symbols $X_{k}, k \in \Omega_{A}$, and then the time-domain signal with a $\mathrm{CP}$ is formed to be sent on the radio channel. Here, $M$ denotes the number of bits in one data symbol. Figure 2 also illustrated two different receiver options for implementing the proposed clipping compensation algorithm. In other words, the purpose of Figure 2 is to illustrate the proposed receiver structures in a simplified manner. It does not present system-level hierarchy, where there are several users and, therefore, several transmitters and receivers. However, the proposed clipping 

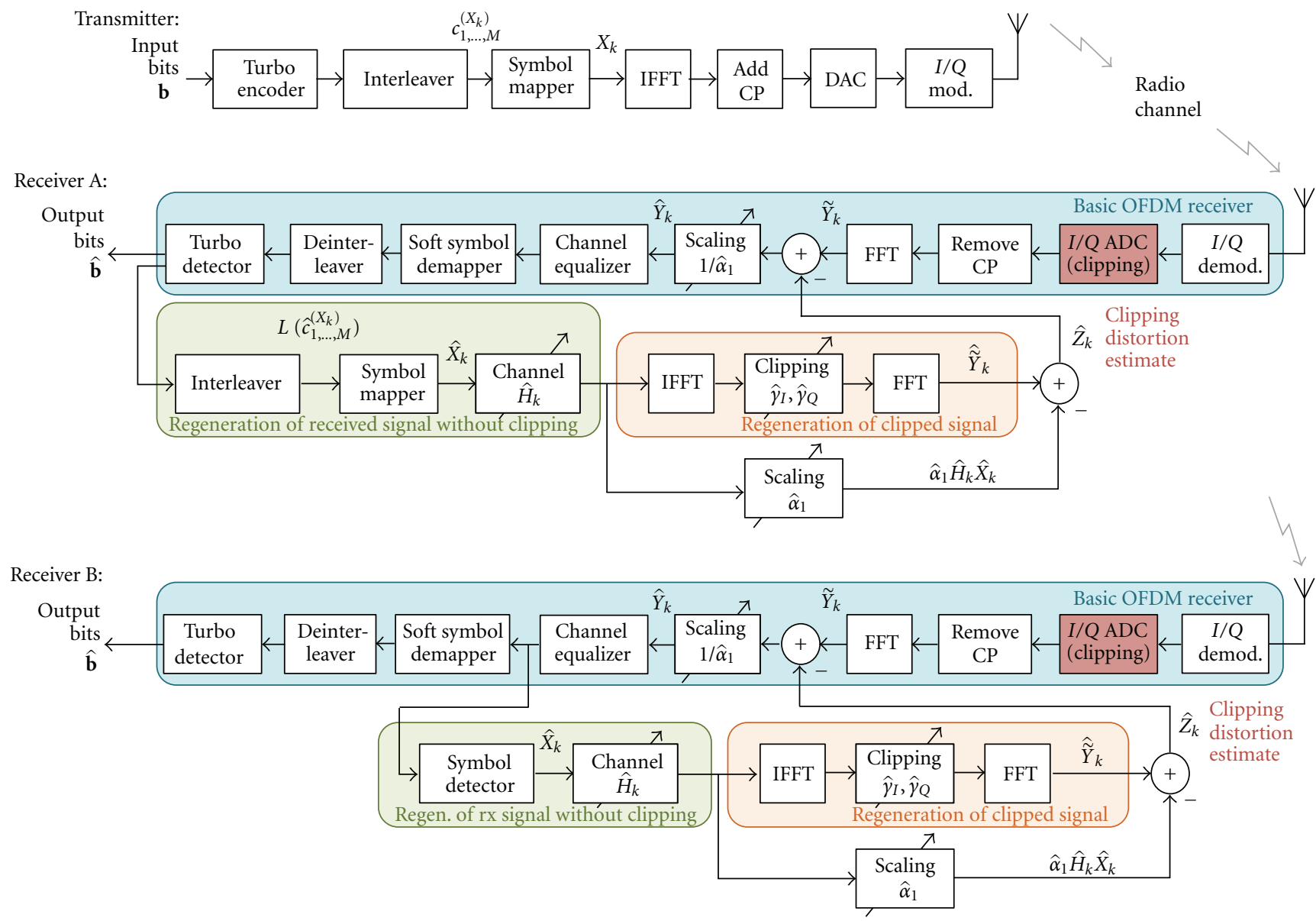

FIGURE 2: Undesired clipping distortion is caused by the A/D converter on the receiver side. Block diagrams of traditional OFDM transmitter and two alternative receivers implementing the proposed clipping compensation algorithm are illustrated. In Receiver A, bits are decoded on every compensation iteration round whereas Receiver B first iterates the clipping compensation on symbol level and only in the end decodes the information bits.

compensation algorithm is able to jointly process multiuser data inside one receiver as is discussed in Section 3.3.

Receiver A in Figure 2 presents the first proposition of this paper on how to remove the clipping distortion caused by the A/D interface of the receiver. In brief, the received and digitized signal is first detected in a conventional manner, that is, transformed to frequency domain, equalized, mapped to bits, deinterleaved, and decoded. Now, the decoded bits are exploited in the clipping compensation feedback loop by generating an estimate of the received signal with and without clipping. Based on (6), an estimate of the clipping distortion in the original received signal can be obtained by subtracting the scaled nonclipped estimate of the received signal from the clipped one. After that, the clipping distortion estimate can be subtracted from the original received signal in order to enhance it and obtain better detection results. Then, the whole process is iterated, because a better clipping distortion estimate can be obtained based on the enhanced detection results. Receiver $B$ in Figure 2 implements this same clipping compensation idea, but the symbol demapper, deinterleaver, and turbo decoder are now located outside the clipping compensation loop. Hence, the computational complexity is greatly reduced since the data is kept on symbol level until the end of the clipping compensation iterations and after that the turbo decoder is used only once. The trade-off is that without turbo decoding, there are likely to be more symbol errors and, therefore, the clipping distortion estimate is not as accurate as it could be.

The following paragraphs describe the proposed clipping compensation algorithm in details by providing the exact mathematical notation. The key steps are also summarized in Algorithms 1 and 2 for Receiver A and B, respectively. It is worth noticing that only one OFDM symbol duration is considered in the equations in order to simplify the notation. The clipping compensation can be done separately for each OFDM symbol and hence the simplified notation does not omit any important details. However, in practice, the processing block length may be longer than one OFDM symbol due to the other receiver functions such as channel decoding, deinterleaving, and equalization, but this does not affect the execution of the clipping compensation, which is performed subcarrierwise. 
Initialization: for all $k \in \Omega_{A}$

(1) Set $i=0, \hat{\alpha}_{1}^{(0)}=1$ and $\hat{Z}_{k}^{(0)}=0$

(2) Obtain channel estimates $\hat{H}_{k}^{(i)}$ using signal

$$
\hat{Y}_{k}^{(i)}=\left(1 / \hat{\alpha}_{1}^{(i)}\right)\left(\tilde{Y}_{k}-\hat{Z}_{k}^{(i)}\right)
$$

(3) Channel equalization: $\hat{Y}_{k}^{(i)} / \hat{H}_{k}^{(i)}$

(4) Demap symbols $\hat{Y}_{k}^{(i)} / \hat{H}_{k}^{(i)}$ to bits, deinterleave and decode to obtain output data bits $\hat{\mathbf{b}}^{(i)}$

Iteration: Increase $i$ by one and do the following steps for all $k \in \Omega_{A}$

(5) Create $\hat{X}_{k}^{(i)}$ based on the coded bits $\widehat{c}_{1, \ldots, M}^{\left(X_{k}^{(i-1)}\right)}$ (hard) or $L\left(\widehat{c}_{1, \ldots, M}^{\left(X_{k}^{(i-1)}\right)}\right)$ (soft)

(6) Estimate clipping levels $\hat{\gamma}_{I}^{(i)}$ and $\hat{\gamma}_{Q}^{(i)}$ as well as attenuation factor $\hat{\alpha}_{1}^{(i)}$

(7) Clip $\hat{H}_{k}^{(i-1)} \hat{X}_{k}^{(i)}$ according to $\hat{\gamma}_{I}^{(i)}$ and $\hat{\gamma}_{Q}^{(i)}$ in time domain to produce $\hat{\widetilde{Y}}_{k}^{(i)}$

(8) Calculate clipping distortion estimate $\hat{Z}_{k}^{(i)}=\hat{\widetilde{Y}}_{k}^{(i)}-\hat{\alpha}_{1}^{(i)} \hat{H}_{k}^{(\mathrm{i}-1)} \hat{X}_{k}^{(\mathrm{i})}$

(9) Remove the distortion from the received signal, that is, $\hat{Y}_{k}^{(i)}=\left(1 / \hat{\alpha}_{1}^{(i)}\right)\left(\tilde{Y}_{k}-\hat{Z}_{k}^{(i)}\right)$

(10) Obtain new channel estimates $\hat{H}_{k}^{(i)}$ using signal $\hat{Y}_{k}^{(i)}$

(11) Channel equalization: $\hat{Y}_{k}^{(i)} / \hat{H}_{k}^{(i)}$

(12) Demap symbols $\hat{Y}_{k}^{(i)} / \hat{H}_{k}^{(i)}$ to bits, deinterleave and decode to obtain output data bits $\hat{\mathbf{b}}^{(i)}$

Algorithm 1: Key steps of the proposed receiver clipping compensation algorithm for Receiver A.

Initialization: for all $k \in \Omega_{A}$

(1) Set $i=0, \hat{\alpha}_{1}^{(0)}=1$ and $\hat{Z}_{k}^{(0)}=0$

(2) Obtain channel estimates $\hat{H}_{k}^{(i)}$ using signal $\hat{Y}_{k}^{(i)}=\left(1 / \hat{\alpha}_{1}^{(i)}\right)\left(\tilde{Y}_{k}-\hat{Z}_{k}^{(i)}\right)$

(3) Channel equalization: $\hat{Y}_{k}^{(i)} / \hat{H}_{k}^{(i)}$

Iteration: Increase $i$ by one and do the following steps for all $k \in \Omega_{A}$

(4) Detect $\hat{Y}_{k}^{(i-1)} / \hat{H}_{k}^{(i-1)}$ to obtain symbols $\hat{X}_{k}^{(i)}$

(5) Estimate clipping levels $\hat{\gamma}_{I}^{(i)}$ and $\hat{\gamma}_{Q}^{(i)}$ as well as attenuation factor $\hat{\alpha}_{1}^{(i)}$

(6) Clip $\hat{H}_{k}^{(i-1)} \hat{X}_{k}^{(i)}$ according to $\hat{\gamma}_{I}^{(i)}$ and $\hat{\gamma}_{Q}^{(i)}$ in time domain to produce $\hat{\widetilde{Y}}_{k}^{(i)}$

(7) Calculate clipping distortion estimate $\hat{Z}_{k}^{(i)}=\hat{\tilde{Y}}_{k}^{(i)}-\hat{\alpha}_{1}^{(i)} \hat{H}_{k}^{(i-1)} \hat{X}_{k}^{(i)}$

(8) Remove the distortion from the received signal, that is, $\hat{Y}_{k}^{(i)}=\left(1 / \hat{\alpha}_{1}^{(i)}\right)\left(\tilde{Y}_{k}-\hat{Z}_{k}^{(i)}\right)$

(9) Obtain new channel estimates $\hat{H}_{k}^{(i)}$ using signal $\hat{Y}_{k}^{(i)}$

(10) Channel equalization: $\hat{Y}_{k}^{(i)} / \hat{H}_{k}^{(i)}$

After iteration:

(11) Demap symbols $\hat{Y}_{k}^{(i)} / \hat{H}_{k}^{(i)}$ to bits, deinterleave and decode to obtain output data bits $\hat{\mathbf{b}}^{(i)}$

Algorithm 2: Key steps of the proposed receiver clipping compensation algorithm for Receiver B. 
If the signal is received perfectly without clipping in the $\mathrm{A} / \mathrm{D}$ interface, it can be written as

$$
Y_{k}=H_{k} X_{k}+W_{k}, \quad k \in \Omega
$$

in frequency domain after removing $\mathrm{CP}$ and taking FFT. In (10), $H_{k}$ is the channel gain and $W_{k}$ is the additive white Gaussian noise component for the $k$ th SC. In other words, this equation represents the ideal case for reference. However, if the A/D converter clips the signal, the frequency-domain version of it is then

$$
\begin{aligned}
\tilde{Y}_{k} & =\alpha_{1} Y_{k}+\alpha_{2} Y_{-k}^{*}+D_{k} \\
& =\alpha_{1}\left(H_{k} X_{k}+W_{k}\right)+\alpha_{2}\left(H_{-k}^{*} X_{-k}^{*}+W_{-k}^{*}\right)+D_{k} \\
& =\alpha_{1} H_{k} X_{k}+Z_{k}+\alpha_{1} W_{k}+\alpha_{2} W_{-k}^{*}, \quad k \in \Omega,
\end{aligned}
$$

as can be derived from (6). This signal is also illustrated in Figure 2. In (11), $Z_{k}=\alpha_{2} H_{-k}^{*} X_{-k}^{*}+D_{k}, k \in \Omega$, which essentially means that unequal clipping levels $\gamma_{I}$ and $\gamma_{Q}$ cause mirror-subcarrier interference $\left(\alpha_{2} H_{-k}^{*} X_{-k}^{*}, k \in \Omega\right)$ in addition to the clipping distortion $\left(D_{k}, k \in \Omega\right)$. However, if $\gamma_{I}=\gamma_{Q}$, then $\alpha_{2}=0$ and thus $Z_{k}=D_{k}, k \in \Omega$. Because all the interference in $Z_{k}, k \in \Omega$, stems from the clipping phenomenon, it is called clipping distortion in the rest of the paper. As shown in Figure 2, prior to the clipping compensation, the data symbols $\widetilde{Y}_{k}, k \in \Omega_{A}$ are equalized, mapped to soft bits, deinterleaved, and then decoded. In the channel equalization stage, the channel gain estimates $\hat{H}_{k}, k \in \Omega_{A}$, for active SCs are obtained based on pilot SCs. Section 3.2 contains more discussion about the channel estimation.

In the first step of the clipping compensation, Receiver A uses the bit estimates after the turbo decoder to regenerate the originally transmitted OFDM signal. All the decoded bits may not be correct and, therefore, it is possible that the regenerated signal does not exactly match the transmitted signal. Hence, the regenerated signal is called the estimate of the transmitted signal and it is denoted as $\hat{X}_{k}, k \in \Omega$, in Figure 2. The data bit estimates $\hat{\mathbf{b}}$ are not re-encoded in the clipping compensation feedback loop, but rather the soft-coded bit estimates $L\left(\widehat{c}_{1, \ldots, M}^{\left(X_{k}\right)}\right), k \in \Omega_{A}$, from the turbo decoder output (after interleaving) are used. Here, $\widehat{c}_{1, \ldots, M}^{\left(X_{k}\right)}$ are hard bit estimates related to the symbol $X_{k}$ and $L\left(\widehat{c}_{1, \ldots, M}^{\left(X_{k}\right)}\right)$ are the log-likelihood ratio (LLR) values for the bit estimates $\hat{c}_{1, \ldots, M}^{\left(X_{k}\right)}$. It is possible to generate either hard or soft symbol estimates and hence both options are studied in Section 4. In Receiver B, it is enough to obtain hard symbol estimates using directly the channel equalizer output. This way, the symbol demapping, channel decoding, and symbol mapping process can be avoided during the clipping compensation process. Since channel decoding is usually the most complex process in the receiver chain, significant savings in computational complexity and latency can be achieved if the channel decoder is located outside the clipping compensation loop. From this point onward, both the Receiver A and B proceed in the same way.

The estimate of the transmitted signal is exposed to the estimated channel and then to the estimated clipping process (separately for $I$ and $Q$ branches) in order to obtain a regenerated version of the clipped received signal $\hat{\widetilde{Y}}_{k}, k \in$ $\Omega_{A}$. The clipping process is performed in time domain using the estimated clipping levels $\hat{\gamma}_{I}$ and $\hat{\gamma}_{Q}$, which should be as close as possible to the clipping levels originally occurred in the $\mathrm{A} / \mathrm{D}$ converter during the reception of the signal. The knowledge of the exact clipping levels $\gamma_{I}$ and $\gamma_{Q}$ is not available, but the estimates $\hat{\gamma}_{I}$ and $\hat{\gamma}_{Q}$ can be calculated as shown in (3). Here, the maximum output levels of the A/D converter $V_{0, I}$ and $V_{0, Q}$ are approximated by following the absolute maximum level of the corresponding signal branch after the A/D converter. An estimate of $P_{\mathrm{in}, I}$ and $P_{\mathrm{in}, Q}$ can be calculated from the hard symbol estimates $\hat{X}_{k}, k \in \Omega$. The clipping process performed in time domain essentially produces a signal, which according to Bussgang's theorem can be written in frequency domain as $\hat{\widetilde{Y}}_{k}=\hat{\alpha}_{1} \hat{H}_{k} \hat{X}_{k}+$ $\hat{Z}_{k}, k \in \Omega_{A}$, where $\hat{\alpha}_{1}$ is the estimate of the attenuation factor. Now, the clipping distortion estimate $\hat{Z}_{k}, k \in \Omega_{A}$ can be extracted from $\hat{\widetilde{Y}}_{k}, k \in \Omega_{A}$, by removing the attenuated desired signal part $\hat{\alpha}_{1} \hat{H}_{k} \hat{X}_{k}, k \in \Omega_{A}$, from it. This estimated desired signal can easily be produced from the nonclipped version of the regenerated received signal as illustrated with the lower branch in the clipping compensation loop in Figure 2 (both in Receivers A and B). That is to say, the estimate of the clipping distortion $\hat{Z}_{k}, k \in \Omega_{A}$, is calculated as

$$
\hat{Z}_{k}=\hat{\widetilde{Y}}_{k}-\hat{\alpha}_{1} \hat{H}_{k} \hat{X}_{k}, \quad k \in \Omega_{A},
$$

where $\hat{\alpha}_{1}=\left(\hat{\alpha}_{I}+\hat{\alpha}_{Q}\right) / 2$ is calculated from (8) using $\hat{\gamma}_{I}$ and $\hat{\gamma}_{Q}$. The out-of-band clipping distortion can be ignored and this is easy to do by processing only the active SCs $\Omega_{A}$, as indicated in (12). Now, the last step in clipping compensation is to improve the received signal $\tilde{Y}_{k}, k \in \Omega_{A}$, by removing the estimated clipping distortion $\hat{Z}_{k}, k \in \Omega_{A}$, from it. The compensated signal is expressed as

$$
\widehat{Y}_{k}=\frac{1}{\hat{\alpha}_{1}}\left(\tilde{Y}_{k}-\hat{Z}_{k}\right), \quad k \in \Omega_{A},
$$

and it is used as a starting point for a new processing round, which begins with the channel estimation and equalization.

One way to show the validity of the clipping compensation approach is to substitute (11) into (13). This leads to the equation that expresses the estimate of the received signal without clipping as

$$
\begin{aligned}
\hat{Y}_{k}= & \frac{\alpha_{1}}{\hat{\alpha}_{1}}\left(H_{k} X_{k}+W_{k}\right) \\
& +\frac{1}{\hat{\alpha}_{1}}\left(Z_{k}-\hat{Z}_{k}\right)+\frac{\alpha_{2}}{\hat{\alpha}_{1}} W_{-k}^{*}, \quad k \in \Omega_{A} .
\end{aligned}
$$

If exactly correct estimates $\hat{\alpha}_{1}$ and $\hat{Z}_{k}, k \in \Omega_{A}$, can be achieved, all essential clipping distortion is thus removed. This is straightforward to see from (14), because with the correct estimates, it reduces to the form of (10) plus additional scaled mirror-subcarrier noise $\left(\alpha_{2} / \hat{\alpha}_{1}\right) W_{-k}^{*}, k \in$ $\Omega_{A}$. However, it is impossible to actually produce exactly 
correct clipping estimate, because the noise $W_{k}, k \in \Omega$, gets also clipped in the A/D converter, but the model cannot reproduce the clipping distortion term of the noise.

In practice, it is usually the case that there are errors in the symbol estimates $\hat{X}_{k}, k \in \Omega_{A}$, on the first processing round. This leads to the inaccurate clipping distortion estimate and, therefore, all the distortion is not removed from the received signal on the first round. However, on every processing iteration round the symbol estimates get better since more and more accurate estimate of the clipping distortion can be obtained. In other words, the compensation algorithm works in the desired manner, if high enough portion of the initial symbol estimates are correct. In order to make the proposed algorithm work in the best possible manner, all the estimated parameters should be recalculated on every iteration. This means the estimates of the channel $\hat{H}_{k}, k \in \Omega_{A}$, the clipping levels $\hat{\gamma}_{I}$ and $\hat{\gamma}_{Q}$ as well as the attenuation factor $\hat{\alpha}_{1}$.

The receiver clipping compensation discussed in this paper has similarities with the deliberate transmitterclipping compensation presented, for example, in [9] and [10] as well as to the power amplifier nonlinearity compensation in [13]. However, there are several fundamental differences, which can be analyzed comparing the aforementioned references to the equations shown in Sections 2 and 3 of this paper. Using consistent notation, the received signal under deliberate transmitter clipping can be expressed as $\tilde{Y}_{k}=H_{k}\left(\alpha X_{k}+D_{k}\right)+W_{k}, k \in \Omega$. When compared to (11), it is evident that the role of the channel is different. In the transmitter clipping case, the clipping is applied before transmission and hence the channel estimation in the receiver is less challenging compared to the receiver clipping case. It is shown in Section 4 that the channel estimation is severely interfered in the receiver clipping case, if clipping compensation is not used. Another essential difference is the clipping model itself. In the deliberate transmitter clipping, the amplitude of a complex signal is limited, that is,

$$
|\widetilde{x}(t)|= \begin{cases}|x(t)|, & |x(t)| \leq V_{0}, \\ V_{0}, & |x(t)|>V_{0},\end{cases}
$$

where as the receiver clipping means that the $I$ and $Q$ signal branches are clipped separately as expressed in (2). This means that it is possible in the receiver clipping case that different clipping levels take place in $I$ and $Q$ branches. Nevertheless, since our signal modeling takes it into account, the clipping compensation with unequal clipping levels is possible as verified in Section 4.

3.2. Implementation Details. In this paper, a zero-forcing channel equalizer is used for simplicity. The channel estimates $\hat{H}_{k}, k \in \Omega_{A}$, are obtained using part of the active SCs $\Omega_{A}$, as pilots, which are denoted here as $\Omega_{P}$. These are evenly distributed among the data SCs $\Omega_{D}=\Omega_{A} \backslash \Omega_{P}$ so that every $L$ th SC is a pilot. The channel estimates of pilot SCs are $\hat{H}_{P, k}=\hat{Y}_{k} / X_{k}, k \in \Omega_{P}$, which are exploited to obtain channel estimates for the data SCs using lowpass interpolation. This means that, first, $L-1$ zeros are inserted between every pilot SC channel estimate. Then, a properly designed FIR lowpass interpolation filter is used to obtain the channel estimates for data SCs. The width of the passband of the interpolation filter corresponds to the original data bandwidth and, therefore, the mean-squared error (MSE) of the interpolated SCs is minimized assuming that the pilot SC channel estimates are correct. As the channel estimation is performed in frequency domain, the use of interpolation filter means calculating convolution between the impulse response of the interpolation filter and the zeropadded channel estimates of the pilots in frequency domain.

From the second clipping compensation iteration round onward, also the symbol estimates from the previous iteration round can be exploited in the channel estimation task. Due to the symbol errors, purely data-symbol-based channel estimation may give poorer results than the estimation using pilots. Therefore, a heuristic approach, which combines these two methods, is proposed in this paper. In the combining, the channel estimates are weighted with the corresponding symbol reliabilities $p\left(X_{k}\right), k \in \Omega_{A}$, so that the channel estimates after combining are

$$
\hat{H}_{k}=\frac{p\left(X_{k}\right)}{2} \hat{H}_{D, k}+\left(1-\frac{p\left(X_{k}\right)}{2}\right) \hat{H}_{P, k}, \quad k \in \Omega_{A},
$$

where $\hat{H}_{D, k}=\hat{Y}_{k} / \hat{X}_{k}, k \in \Omega_{D}$ are the channel estimates obtained from the latest data symbol estimates and $\hat{H}_{P, k}, k \in$ $\Omega_{A}$, are the latest channel estimates obtained using pilots. The estimates $\hat{H}_{D, k}, k \in \Omega_{P}$ for pilot SCs are not needed since (16) implicates that $\hat{H}_{k}=\hat{H}_{P, k}, k \in \Omega_{P}$. This is guaranteed by setting $p\left(X_{k}\right)=0, k \in \Omega_{P}$. For the data symbols, the reliabilities are calculated as

$$
p\left(X_{k}\right)=\prod_{j=1}^{M} \frac{\exp \left[\widehat{c}_{j}^{\left(X_{k}\right)} L\left(\hat{c}_{j}^{\left(X_{k}\right)}\right)\right]}{1+\exp \left[L\left(\hat{c}_{j}^{\left(X_{k}\right)}\right)\right]}, \quad k \in \Omega_{D} .
$$

This data-symbol-based estimation is used in Receiver A. Since the LLR values for the bits are not calculated in Receiver B in every iteration, it uses only the pilot-based channel estimation. One variation of the data-symbol-based channel estimation proposed above would be such that a certain threshold is used for the symbol reliabilities. In other words, only the symbols which are reliable enough, let us say $p\left(X_{k}\right)>0.95$, are used in the channel estimation. However, this variation of the proposed channel estimation technique is omitted from the performance analysis of Section 4 and is left for future studies.

The output of the zero-forcing channel equalizer should be limited so that the maximum amplitude is

$$
V_{\text {max }, \mathrm{eq}}=\max _{r}\left\|A_{r}\right\|+\frac{3.29}{\sqrt{2}} \sigma_{n},
$$

where $A_{r}, r=1, \ldots, 2^{M}$ denotes all the possible symbols from the used alphabet and $\sigma_{n}$ is the standard deviation of the complex noise $w(t)$, which is assumed to be Gaussian. The factor of 3.29 corresponds to the $99.9 \%$ confidence level of one-dimensional Gaussian distribution and, therefore, the standard deviation of the complex noise has to be scaled with $\sqrt{2}$. The amplitude limit of the equalizer output 
does not have to be exactly what is proposed in (18), but significantly too tight or loose limit affects decoding results. The reason to use such an amplitude limiter after the channel equalizer is the soft symbol demapper defined based on an assumption of Gaussian noise. The clipping distortion and the channel equalizer cause interference peaks, which degrade the performance of the soft symbol demapper. In this paper, it is chosen to set the LLR values of the bits related to the amplitude-limited symbols to zero. This is justified, because if there is a significant distortion term present in a symbol, all the usable information carried by the symbol has already been lost. The main target in the limiting of the equalizer output is to tell the decoder which symbols are very unreliable due to the channel fading or unintentional clipping, but in the same time avoid affecting the symbols which are spread only because of the Gaussian noise.

The channel codec used in this paper is a turbo codec [20] with a generator matrix:

$$
G=\left[\begin{array}{ll}
1 & 15 \\
13
\end{array}\right] .
$$

The used interleavers are bit-wise S-interleavers [21] for which the distance parameter is defined as $S=\sqrt{U / 2}$, where $U$ is the length of the unit to be interleaved. In channel interleaving, the unit is the whole coded block $\left(U_{\text {out }}\right)$. Inside the turbo encoder/decoder, the length of the interleaved unit is equal to one uncoded data block $\left(U_{\text {in }}\right)$. This is defined as $U_{\text {in }}=\left\lfloor R\left(U_{\text {out }}-2 m\right)\right\rfloor$, where $m=3$, is the memory length of the component encoder and the term $2 m$ is due to the unpunctured termination bits [22]. Different coding rates are achieved by puncturing the parity bits, which is done based on [22]. The turbo decoder uses the Max-Log-MAP algorithm for decoding, and it does not have a correction function for the max-operator [23]. The iteration of turbo decoding is stopped based on the hard-data-aided (HDA) criterion presented in [24].

3.3. Multiuser Considerations. Since the proposed receiver clipping compensation method is very general, it can be used both in UL and DL directions. However, there are some essential differences when a multiuser scenario is concerned. In DL direction, the data symbol sequence $X_{k}, k \in \Omega_{A}$, contains symbols, which belong to several individual mobile users. A single mobile receiver should be able to process, in addition to its own data, also the symbols of neighboring users in order to exploit the clipping compensation method in an optimal manner. This is because of the nonlinear behavior of clipping, which causes that part of the clipping distortion is stemming from outside the frequency band of the user. If the signal power for the neighboring users is higher than for the interested user, the amount of clipping distortion can be very high. Therefore, joint clipping compensation processing of all neighboring users is preferable. The challenge in the implementation of the clipping compensation in mobile receiver is that the amount of signal processing is significantly increased when the symbols of neighboring users are processed and the channel estimation is performed over the whole frequency band instead of concentrating on one user only as the mobile receiver usually does. The proposed Receiver $\mathrm{B}$ model is especially suitable for DL direction due to its considerably lower computational complexity but still relatively good performance, as shown in Section 4.1. One way to decrease the computational burden even more is to exploit cooperative interference cancellation among the users so that they exchange information of their own data and interference using short-range communications. For example, spatial correlation of the channels can be exploited, if users are close to each other.

Similarly as in the DL direction, also in the UL scenario the data symbol sequence $X_{k}, k \in \Omega_{A}$, contains data of multiple users. Furthermore, in UL, a band-wise channel estimation for each user must be performed individually, since the different mobile signals have propagated through different channels. Therefore, the channel estimate sequence $\hat{H}_{k}, k \in \Omega_{A}$, is not continuous. In general, the implementation of the clipping compensation method is more viable in the UL scenario since the base station has to receive and process the signals of all users anyway. Hence, including the clipping compensation processing is not going to excessively increase the computational complexity. On the other hand, modern base stations can also have vast amount of computing power, which makes complex digital signal processing algorithms feasible. Due to its higher computational complexity, Receiver A model is proposed for the UL direction. More discussion of the receiver complexity and performance can be found in Section 4.1.

\section{Performance Results for the Clipping Compensation Approach}

In this section, the performance of the proposed receiverside clipping compensation algorithm is analyzed and discussed in details. This is done using a UL scenario with two individual users in the block-fading extended ITU-R Vehicular A channel [25]. First, Section 4.1 analyzes the performance of the clipping compensation algorithm through computer simulations. After that, Section 4.2 considers the same scenario using laboratory measurements with real A/D hardware. Finally, Section 4.3 presents analysis of computational complexity for Receivers $\mathrm{A}$ and $\mathrm{B}$ in the considered scenario.

Since an UL scenario is considered, both users experience different realizations of the fading channel. Here, it is also assumed that the channels stays static for ten consecutive OFDM symbols, which makes possible to average obtained channel estimates over this period. The channel conditions are set so that the average received power level for User 1 is $15 \mathrm{~dB}$ less than for User 2. In this section, it is assumed that there is perfect delay and frequency alignment between the users. The most essential parameters of the simulated scenario are collected into Table 1 . Both users have 512 active SCs of which every eighth is a pilot $(L=8)$. Therefore, $N_{A}=1024$ in the receiver side, when the composite signal of two users is processed. The pilot power is adjusted so that it corresponds to the average power of the data symbols. 
TABLE 1: Parameters for the simulated uplink multiuser scenario.

\begin{tabular}{lc}
\hline Parameter & Value \\
\hline Number of users & 2 \\
FFT size $N$ & 2048 \\
Active subcarriers per user & 512 \\
Scattered pilots per user & 64 \\
CP length & 128 samples \\
Sampling rate & $30.72 \mathrm{MHz}$ \\
Subcarrier spacing & $15 \mathrm{kHz}$ \\
Subcarrier modulation & $16-\mathrm{QAM}(M=4)$ \\
Channel profile & Extended ITU-R Veh. A [25] \\
Channel codec & Turbo codec (Max-Log-MAP) \\
Coding rate $R$ & $1 / 2$ \\
Coded block length $U_{\text {out }}$ & 3584 bits (2 OFDM symbols) \\
Decoding iterations & Max. 5 \\
Clipping comp. iterations $I$ & 5 (or 10) \\
Clipping level $\gamma$ & $0-10 \mathrm{~dB}$ \\
SNR for User $1 S N R_{1}$ & $16-36 \mathrm{~dB}$ \\
\hline
\end{tabular}

Both users have 16-QAM as an SC modulation and hence $M=4$. The oversampling factor used is $J=2$ and the overall sampling rate is $30.72 \mathrm{MHz}$, which results in the SC spacing of $15 \mathrm{kHz}$. The length of $\mathrm{CP}$ is $(1 / 16) N=128$ samples. On average, the PAPR of the composite signal of two users is $10.1 \mathrm{~dB}$. For the channel coding, the turbo codec is used and its coded block length is $\mathrm{U}_{\text {out }}=3584$ bits, meaning that two consecutive OFDM symbols form one coded block. Coding rate of $R=1 / 2$ is used and hence $\mathrm{U}_{\text {in }}=1789$ bits. In every clipping compensation iteration, the turbo decoder is allowed to do five decoding iterations, if not stopped earlier based on the HDA criterion. In the following, five clipping compensation iterations $(I=5)$ have been performed in all cases, if not specifically stated otherwise. In practical receiver, unnecessary iterations can be avoided, to decrease the amount of computation, by stopping iterations for the user, if its bit-error ratio (BER) goes to zero before the maximum amount of iterations has been performed. This can be checked, for example, by exploiting the cyclic redundancy check (CRC).

4.1. Simulation Results. Figure 3 gives an overall illustration of the above-defined scenario, when the clipping level $\gamma=$ $6 \mathrm{~dB}$ occurs. For simplicity, equal clipping levels $\gamma_{\mathrm{I}}=$ $\gamma_{\mathrm{Q}}=\gamma$ are assumed in this section, if not otherwise stated. Figure 3(a) shows a piece of the real branch signal before and after clipping. Correspondingly, Figure 3(b) illustrates the received waveform in frequency domain before and after clipping. It is clearly visible that the weak User 1 suffers from the nonlinear clipping distortion stemming from the band of the considerably stronger User 2.

The progress of the clipping compensation process for User 1 is illustrated in Figure 4 when Receiver A is used for the case shown in Figure 3. The BER results are averaged over 20,000 OFDM symbols, that is, 2000 independent channel

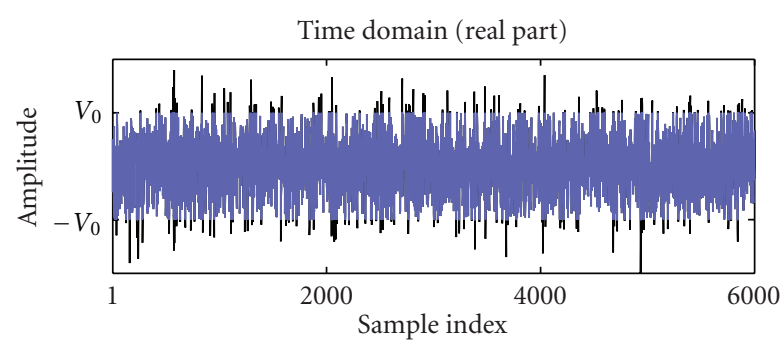

(a)

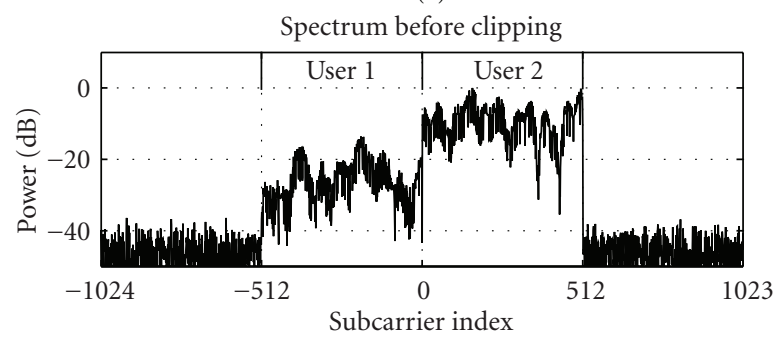

(b)

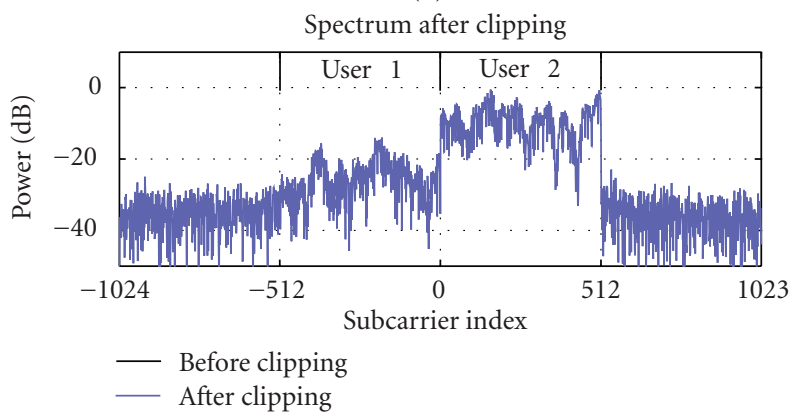

(c)

Figure 3: (a) Time domain illustration of the real part of the received signal before and after clipping, $\gamma=6 \mathrm{~dB}$ (b) spectrum of the received signal before clipping (c) spectrum of the signal after clipping.

realizations, as is done also for all the other performance figures in this Section. In this paper, BER is calculated for the data bits $\mathbf{b}$. Figure 4 shows that the proposed clipping compensation algorithm is able to almost reach the BER of the nonclipped signal, which represents here the error floor. For the nonclipped signal, perfect channel knowledge is used in order to present the ideal reference case whereas the channel estimation proposed in Section 3.2 is used in the clipping compensation. Figure 4 also illustrates the information flow inside the receiver. Decoding arrow (D) refers to the turbo decoding process and feedback arrow (F) denotes the process where bit decisions are exploited to remove clipping distortion from the received signal. It can be seen from Figure 4 that the turbo decoder cannot reduce the BER of the noncompensated signal very much due to the significant amount of clipping distortion. Nonetheless, the clipping compensation method is still able to reduce the amount of distortion, and in the next iteration, the turbo decoder reduces the BER more. This means that the clipping compensation performs well even without turbo decoding and thus the Receiver B model is justified. 


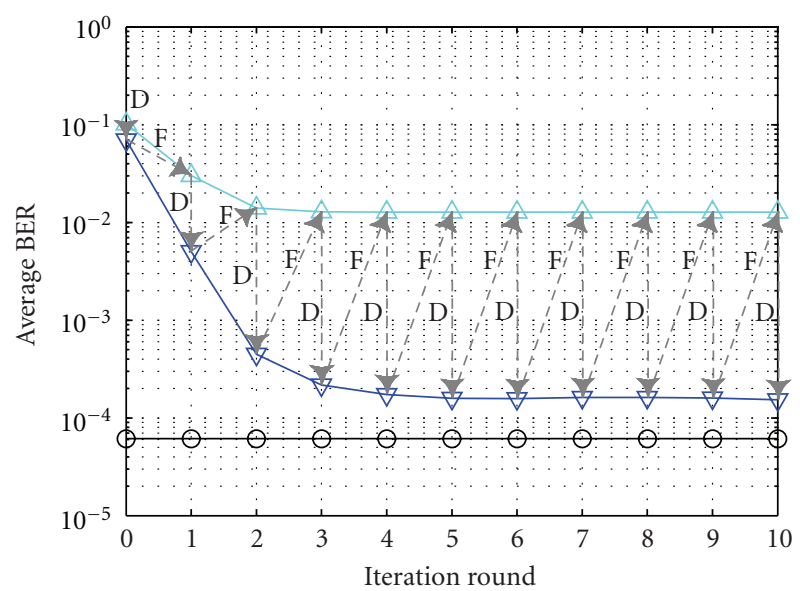

(a)

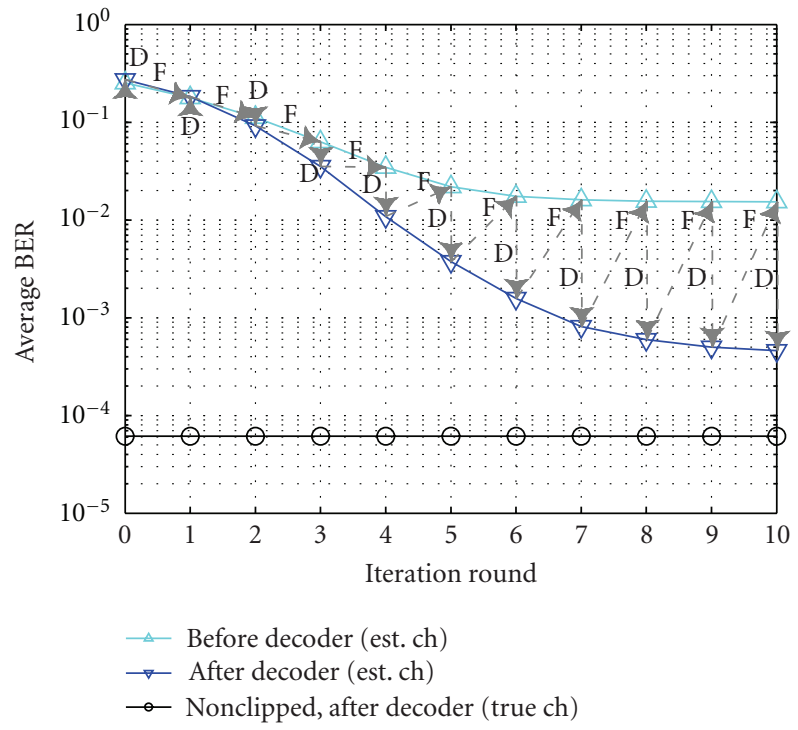

(b)

Figure 4: Average BER as a function of clipping compensation iterations for User 1 using Receiver A when $S N R_{1}=21.9 \mathrm{~dB}$ and (a) $\gamma=6 \mathrm{~dB}$ or (b) $\gamma=2 \mathrm{~dB}$. Iteration round 0 corresponds to the noncompensated signal. Arrows refers to the decoding (D) and compensation feedback (F) stages.

A detailed performance comparison between Receiver A and $\mathrm{B}$ is given later in this Section. For the results shown in Figure 4, the average received signal-to-noise ratio $(S N R)$ for User 1 is $21.9 \mathrm{~dB}$ whereas for User 2 it is $36.9 \mathrm{~dB}$. Here, the average received $S N R$ is defined using the average signal and noise powers on the user frequency band, that is,

$$
S N R_{u}=\frac{E\left[\sum_{k \in \Omega_{A, u}}\left|H_{k} X_{k}\right|^{2}\right]}{E\left[\sum_{k \in \Omega_{A, u}}\left|W_{k}\right|^{2}\right]},
$$

where $u$ is the user index, $\Omega_{A, 1}=\left\{-N_{A} / 2, \ldots,-1\right\}$ for User 1 and $\Omega_{A, 2}=\left\{1, \ldots, N_{A} / 2\right\}$ for User 2. By comparing Figures $4(\mathrm{a})$ and $4(\mathrm{~b})$, it can be concluded that stronger clipping requires more compensation iterations rounds, but eventually most of the clipping distortion is removed.
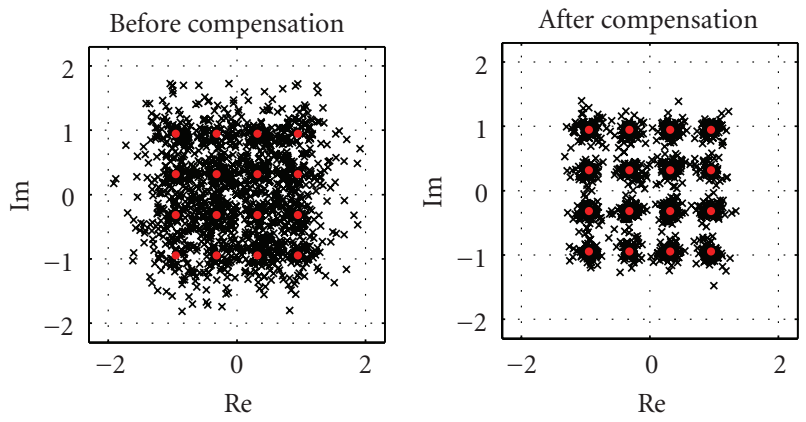

FIGURE 5: Example realization of the received constellation for User 1 before and after clipping compensation in Receiver A when $\gamma=$ $6 \mathrm{~dB}$ and $S N R_{1}=21.9 \mathrm{~dB}$.

An example of the received constellation before and after the clipping compensation for User 1 is provided in Figure 5 to visually illustrate the reduction of clipping distortion due to the applied compensation method. This matches with the cases shown in Figures 3 and 4 . In addition to BER results, one way to illustrate the performance of the clipping compensation method is the subcarrier-wise signalto-clipping-distortion ratio $(S C D R)$, which is defined as

$$
\operatorname{SCDR}_{k}=\frac{E\left[\left|Y_{k}\right|^{2}\right]}{E\left[\left|\hat{Y}_{k}-Y_{k}\right|^{2}\right]}, \quad k \in \Omega_{A} .
$$

It describes how much there is clipping distortion compared to the ideal nonclipped situation. Figure 6 shows SCDR results for both users when $\gamma=6 \mathrm{~dB}$, which is the same case that is considered in Figures 3-5. It can be seen from Figure 6 that in this particular case the amount of clipping distortion is decreased over $20 \mathrm{~dB}$ for both users. It should be kept in mind that SCDR values are relative. User 1 has considerably more clipping distortion than User 2 in the beginning and hence the improvement in SCDR has much more significant effect for User 1. Naturally, the employed channel estimation approach has a finite accuracy, but this is a minor matter compared to the fact that the receiver-side clipping affects the pilots and, therefore, notably distorts the channel estimates. On the other hand, the clipping compensation also effectively enhances the channel estimates. The subcarrierwise channel estimation MSE is given in Figure 7 for the currently discussed case of $\gamma=6 \mathrm{~dB}$. This figure verifies that the clipping compensation without a doubt improves the channel estimates. The channel estimation MSE for the nonclipped signal in Figure 7 illustrates the error floor for this particular channel estimation method and noise level. The relatively high error on the sides of the user bands is stemming from the interpolation filter transients.

In order to provide a wider view of the clipping compensation performance, Figure 8 illustrates the simulated BER results for User 1 as a function of occurred clipping level when $S N R_{1}=21.9 \mathrm{~dB}$. The same figure also provides a comparison between the proposed channel estimation and perfect channel knowledge. Although the channel estimation works well when $\gamma>4 \mathrm{~dB}$, the channel estimates gets 


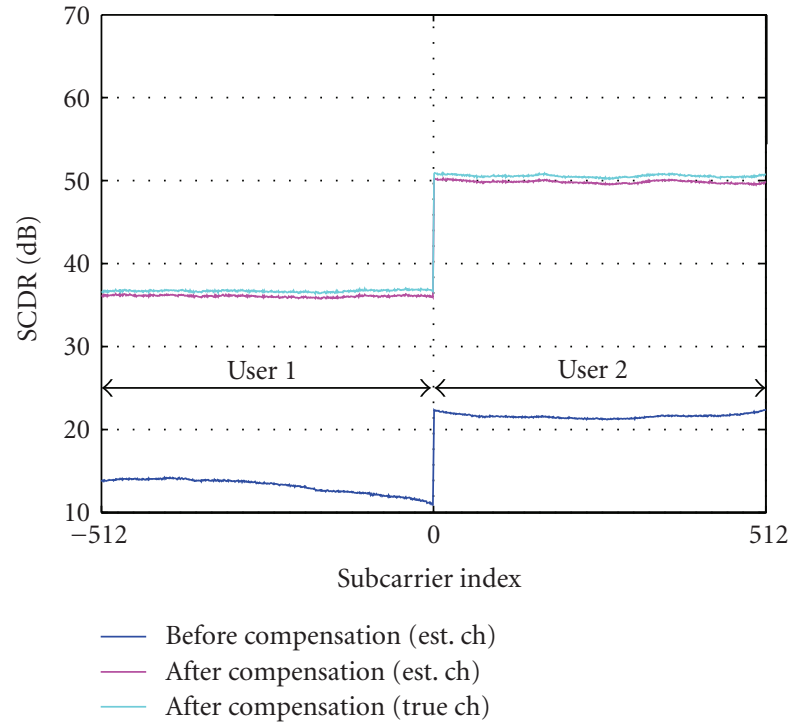

FIGURE 6: Subcarrier-wise signal-to-clipping-distortion ratio when $\gamma=6 \mathrm{~dB}, S N R_{1}=21.9 \mathrm{~dB}$ and $S N R_{2}=36.9 \mathrm{~dB}$. The results are obtained after 5 clipping compensation iterations using Receiver A.

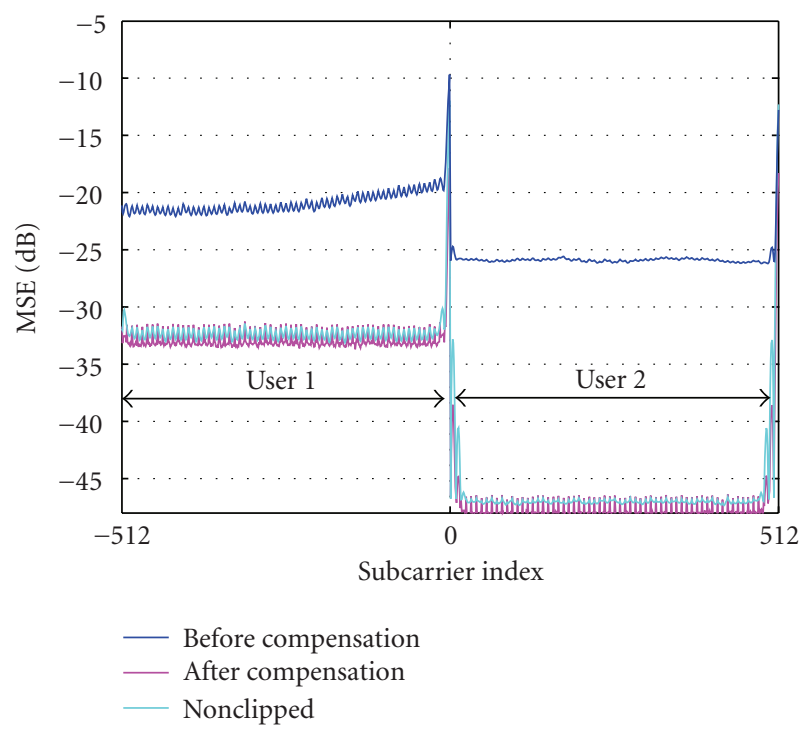

FIGURE 7: Mean squared error of channel estimates when $\gamma=6 \mathrm{~dB}$, $S N R_{1}=21.9 \mathrm{~dB}$ and $S N R_{2}=36.9 \mathrm{~dB}$. The results are obtained after 5 clipping compensation iterations using Receiver $\mathrm{A}$.

significantly poorer if heavier clipping occurs. Because the input $S N R$ is the same for all clipping levels, it can be concluded that the channel estimation accuracy is limited due to the clipping distortion and not because of the noise. These results were obtained after five clipping compensation iterations, which does not guarantee that BER is always minimized. Under heavy clipping, additional iterations would provide lower BER to some extent, but there is always a certain error floor despite the number of iterations. Another support for this issue is provided in Figure 9, where the

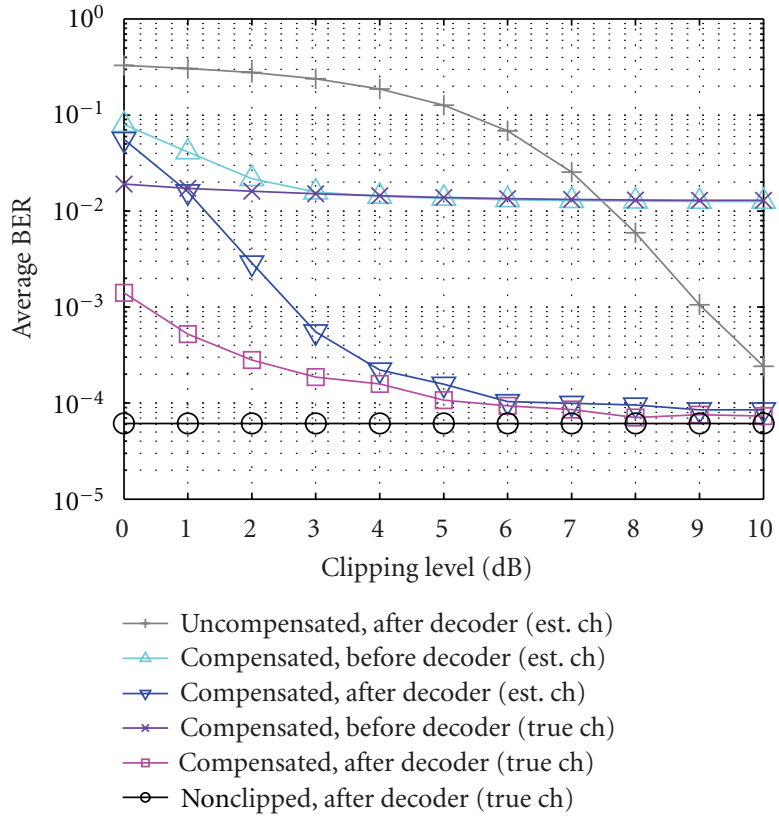

FIGURE 8: Performance of the clipping compensation for User 1 as a function of clipping level when $S N R_{1}=21.9 \mathrm{~dB}$. The results are obtained after 5 clipping compensation iterations using Receiver A.

BER of User 1 is illustrated as a function $S N R_{1}$ for clipping levels $\gamma=6 \mathrm{~dB}$ and $\gamma=2 \mathrm{~dB}$. From here, it can be seen that the clipping compensation performs decently, if the noise level and the clipping level are reasonable. The limited channel estimation accuracy due to the heavy clipping can be seen in Figure 9(b) by comparing the BER curves in cases of estimated channel and perfect channel knowledge. This means that the clipping compensation method is able to recover the BER almost to the level of nonclipped signal even under heavy clipping, if the channel estimation could be performed accurately. The limitations of the channel estimation can be relieved to some extend by increasing the number of compensation iterations, but in order to minimize the number of iterations, the optimal channel estimation approach under clipping is an interesting topic for future research. Figure 10 shows the BER of User 1 separately for every clipping compensation iteration round when $\gamma=6 \mathrm{~dB}$. From here, it can be concluded that almost the maximum performance is achieved within only three iterations. The rapid saturation of the performance is also observable from Figure 4(a) whereas stronger clipping would require a few iterations more. It is desirable to minimize the number of iterations since it directly affects the processing time of the clipping compensation per code block and thus also the overall latency of the receiver.

One interesting question: does the performance of the clipping compensation method change if soft symbol estimates are used instead of the hard ones. Figures 11 and 12 show that the performance for User 1 using Receiver A is practically the same for hard and soft symbol estimates. It is worth clarifying that the soft symbol estimates are used only for the regeneration of the transmitted signal 


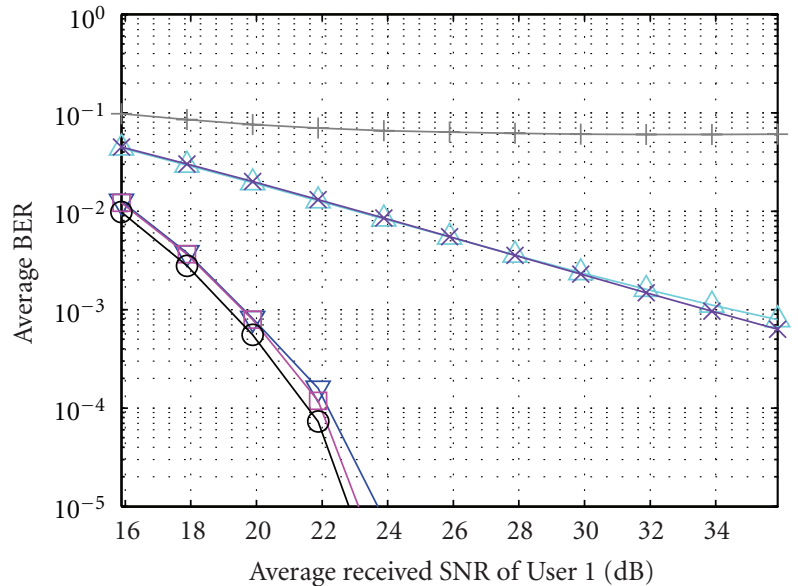

(a)

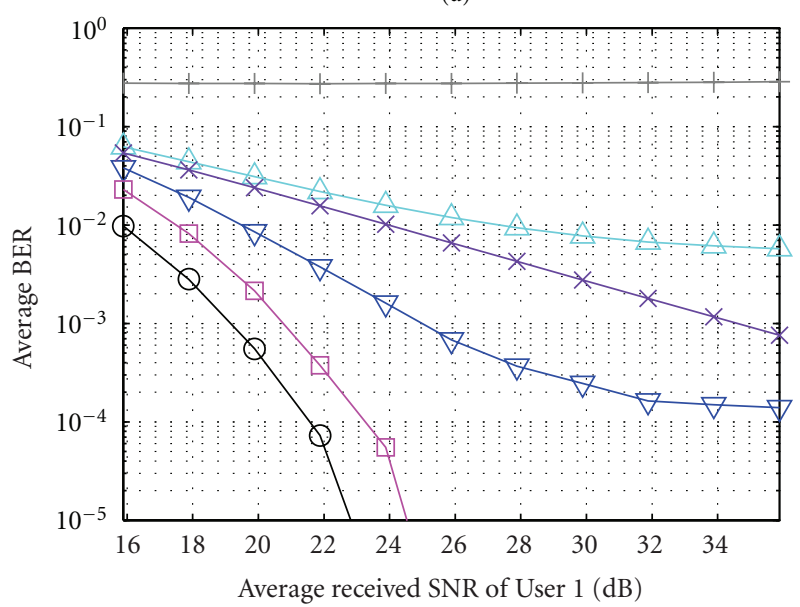

- Uncompensated, after decoder (est. ch)

$\triangle$ Compensated, before decoder (est. ch)

$\rightarrow$ Compensated, after decoder (est. ch)

$\rightarrow$ Compensated, before decoder (true ch)

$\rightarrow$ Compensated, after decoder (true ch)

$\rightarrow$ Nonclipped, after decoder (true ch)

(b)

FIGURE 9: Performance of the clipping compensation for User 1 as a function of average received $S N R$ for clipping levels (a) $6 \mathrm{~dB}$ and (b) $2 \mathrm{~dB}$. The results are obtained after 5 clipping compensation iterations using Receiver A.

in the compensation feedback loop. The channel estimates $\hat{H}_{D, k}, k \in \Omega_{D}$, and the estimate of $P_{\text {in }}$ are always obtained using the hard symbol estimates, because the soft symbol estimates would give misleading results. For a deliberate transmitter clipping compensation, it is reported in [10] that the soft symbol estimates lead to equal or even worse performance compared to the hard symbol estimates. Based on Figures 11 and 12, it can be stated that the hard symbol estimates should be used in Receiver A, since the soft symbol estimates would only unnecessarily increase the computational load. Another comparison made in Figures 11 and 12 is between Receivers A and B. Even though the computational complexity of Receiver B is significantly

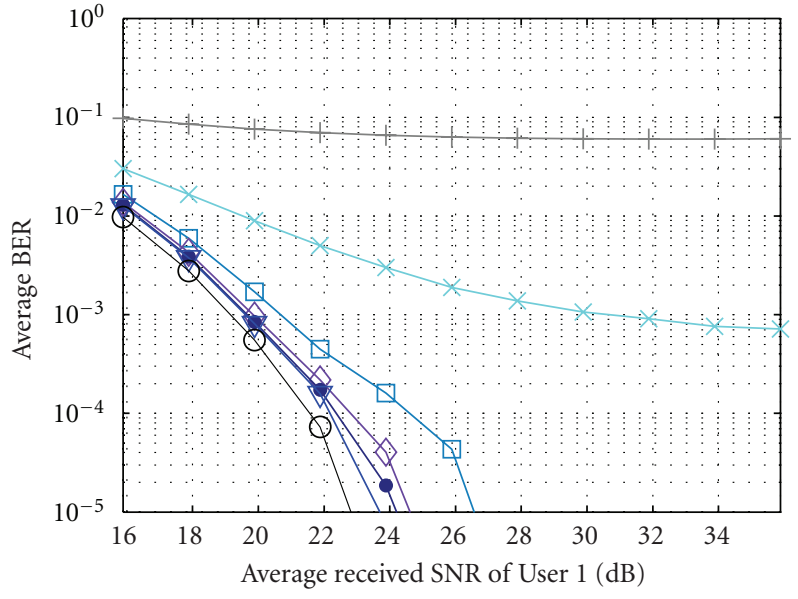

$\longrightarrow$ Uncompensated, after decoder (est. ch)
- Compensated iteration 1, after decoder (est. ch)
$\square-$ Compensated iteration 2, after decoder (est. ch)
$\rightarrow$ Compensated iteration 3, after decoder (est. ch)
$\rightarrow-$ Compensated iteration 4, after decoder (est. ch)
$\rightarrow$ Compensated iteration 5, after decoder (est. ch)
$\rightarrow$ Nonclipped, after decoder (true ch)

FIgURE 10: Average BER of User 1 separately for every clipping compensation iteration round as a function of average received $S N R$ when Receiver $\mathrm{A}$ is used and $\gamma=6 \mathrm{~dB}$.

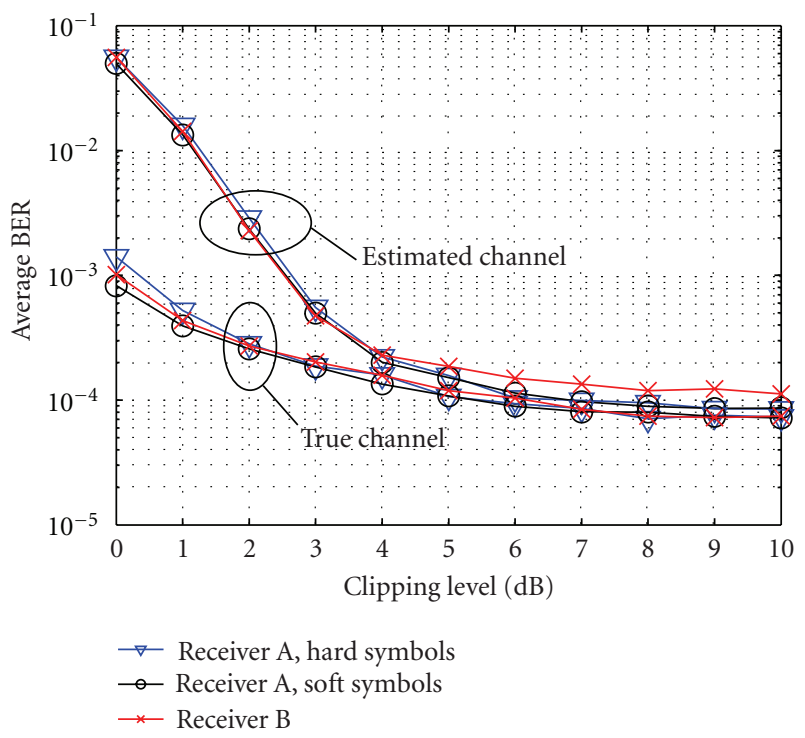

Figure 11: Performance comparison between Receivers A and B for User 1 as a function of clipping level when $S N R_{1}=21.9 \mathrm{~dB}$ and 5 compensation iterations is performed.

lower, it performs only marginally worse than Receiver A with all the clipping levels and SNRs studied. Therefore, the Receiver B model is suggested especially for mobile receivers. In practice, one option would be to consider a hybrid of Receiver A and B. This means first iterating the clipping compensation on the symbol level until the performance saturates, then perform the turbo decoding, and finally do 


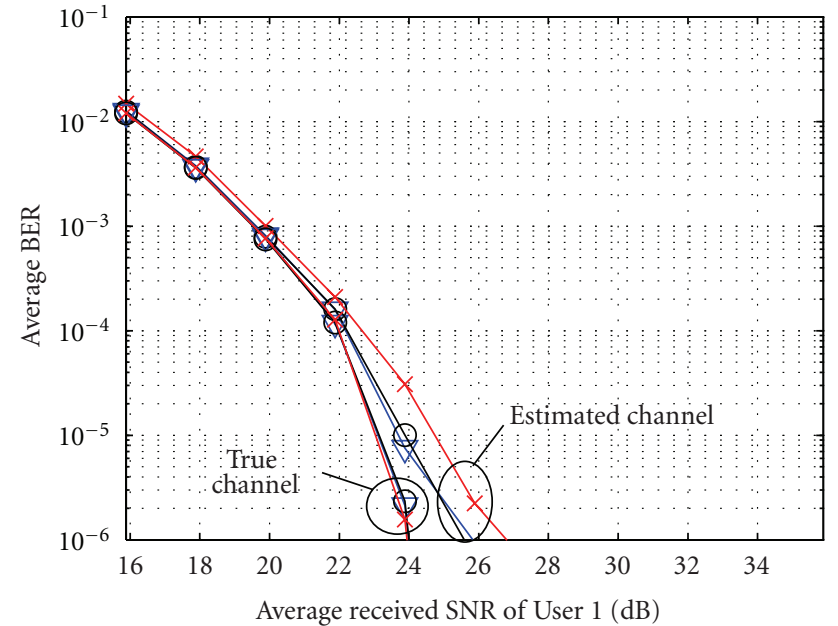

(a)

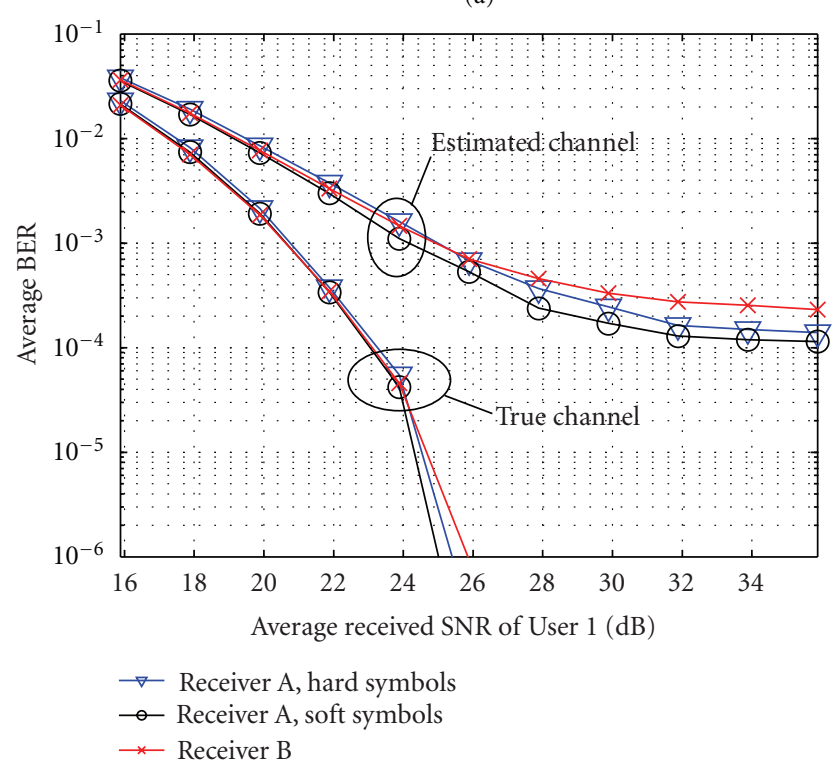

(b)

FIgure 12: Performance comparison between Receivers A and B for User 1 as a function of average received SNR when 5 compensation iterations is performed and clipping level is (a) $6 \mathrm{~dB}$ or (b) $2 \mathrm{~dB}$.

one more clipping compensation iteration as well as the final turbo decoding. This would provide a compromise between the performance and computational complexity.

Generally in this section, equal clipping levels in $I$ and $Q$ branches are assumed. However, the proposed clipping compensation algorithm completely supports unequal clipping levels as discussed in Sections 2 and 3. This feature is desired, since in practice there can be slight differences between the A/D converters of $I$ and $Q$ branch. In addition, other imbalances of analog circuitry can also affect the clipping levels. Figure 13 presents BER results for a clipping compensation example, where $\gamma_{I}=6 \mathrm{~dB}$ and $\gamma_{\mathrm{Q}}=3 \mathrm{~dB}$. The 3-dB difference in clipping levels can be considered to be a rather extreme situation but is used for demonstration purposes. However, the clipping compensation algorithm is

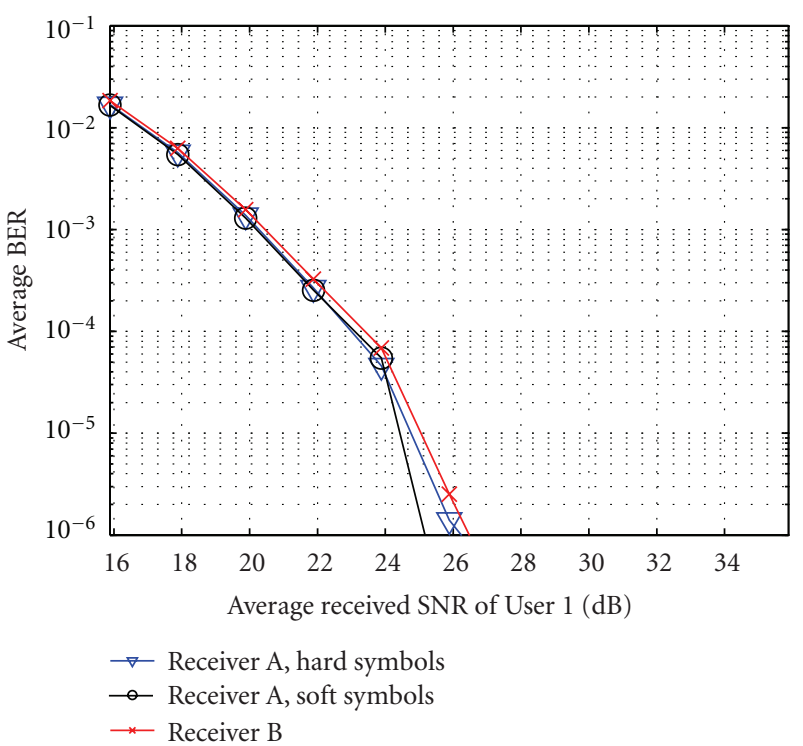

Figure 13: Performance comparison between Receivers A and B with channel estimation for User 1 as a function of average received SNR when 5 compensation iterations is performed and the clipping levels for $I$ and $Q$ branches are $\gamma_{I}=6 \mathrm{~dB}$ and $\gamma_{Q}=3 \mathrm{~dB}$.

able to perform very well as can be seen from Figure 13 . There is only a slight performance decrease compared to the Figure 12(a), where equal clipping levels $(\gamma=6 \mathrm{~dB})$ are assumed. The small performance decrease is expected, because stronger clipping is more challenging to compensate as was shown in Figure 8.

4.2. Laboratory Measurement Experiment. The same twouser scenario used in the simulations of the previous Section is also used in laboratory measurements in order to provide comparable results. Only difference is that 10,000 OFDM symbols (instead of 20,000) are used in averaging the BER results due to the slowness of performing the measurements. The complete measurement setup is illustrated in Figure 14. First, a composite waveform of two users is created with a PC. Also the fading channel model is applied to the waveform already on the PC and noise is added so that the $S N R$ of the overall waveform is $31 \mathrm{~dB}$. This corresponds to the case used in the simulations, where $S N R_{1}=21.9 \mathrm{~dB}$ and $S N R_{2}=$ $36.9 \mathrm{~dB}$ were calculated. This digital waveform is then sent to the baseband signal generator [26] in order to convert it to the analog domain. The communication interface between the PC and the signal generator is implemented with TCP/IP. The typical spurious free dynamic range of the used signal generator with a 14-bit D/A converter is $83 \mathrm{dBc}$ [26]. Therefore, it can be assumed that the signal generator does not significantly change the generated waveform. The analog waveform is then fed to the 14-bit A/D converter [27] using balanced coaxial cable connection. The output voltage of the signal generator is adjusted so that it exceeds the maximum voltage level of the $\mathrm{A} / \mathrm{D}$ converter input and the waveform gets clipped. The digitized clipped waveform is then read from the buffer memory of the $\mathrm{A} / \mathrm{D}$ converter 


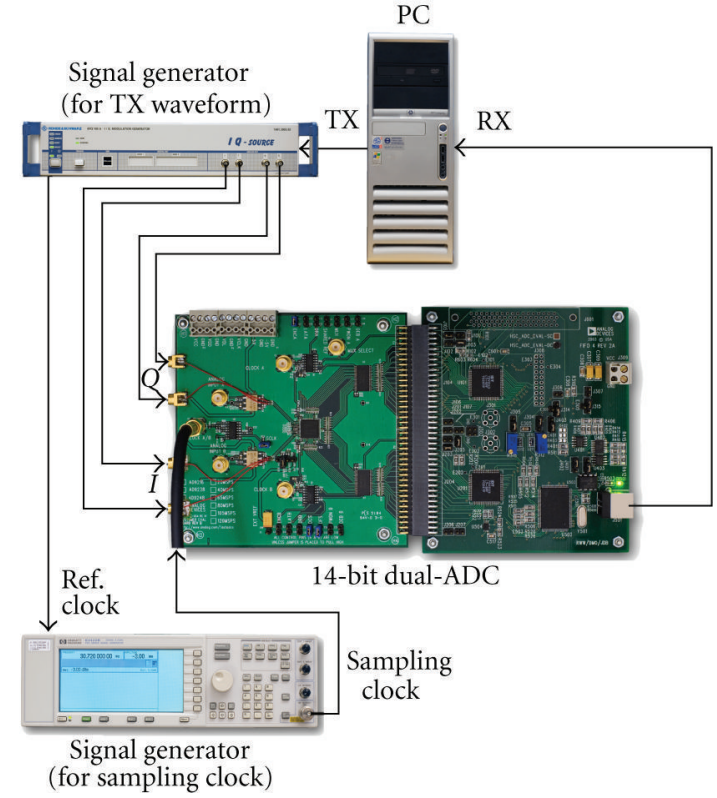

FIgURE 14: Laboratory measurement set-up. A signal waveform with fading channel is generated using the PC and fed to the baseband signal generator [26] to create an analog waveform. The output power of the signal generator is adjusted so that the signal is clipped in the $A / D$ converter [27]. The digitized signal is then collected to the PC for clipping compensation.

board to the PC using USB interface. Finally, the PC applies the proposed iterative clipping compensation method to the clipped waveform. The other signal generator shown on the lower left corner of Figure 14 is used to feed sampling clock signal for the $\mathrm{A} / \mathrm{D}$ converter. The sampling rate is $30.72 \mathrm{MHz}$ as specified in Table 1. There is also a reference clock signal between the signal generators to synchronize their internal oscillators.

The amount of noise and distortion the measurement setup creates can be obtained by running the measurements with a low voltage level so that the waveform does not clip in the A/D converter. The estimated SNR after measurements for the overall nonclipped waveform was $30.87 \mathrm{~dB}$. In order to take also the distortion into account, the signal-to-noiseand-distortion ratio $(S N D R)$ for the frequency band of user $u$ is defined as

$$
\operatorname{SNDR}_{u}=\frac{E\left[\sum_{k \in \Omega_{A, u}}\left|H_{k} X_{k}\right|^{2}\right]}{E\left[\sum_{k \in \Omega_{A, u}}\left|\tilde{Y}_{k}-H_{k} X_{k}\right|^{2}\right]} .
$$

Using the measured nonclipped waveform, it was observed that $S N D R_{1}=21.7 \mathrm{~dB}$ and $S N D R_{2}=36.5 \mathrm{~dB}$. Figure 15 illustrates the clipping compensation results for User 1 in the laboratory measurement cases in which the occurred clipping is $5-10 \mathrm{~dB}$. These are average estimated clipping levels for certain output voltage levels of the signal generator since the exact knowledge of clipping levels is not available. By comparing the BERs before and after the clipping compensation in Figure 15, it can be concluded that considerable gain is obtained. In addition, the performance difference

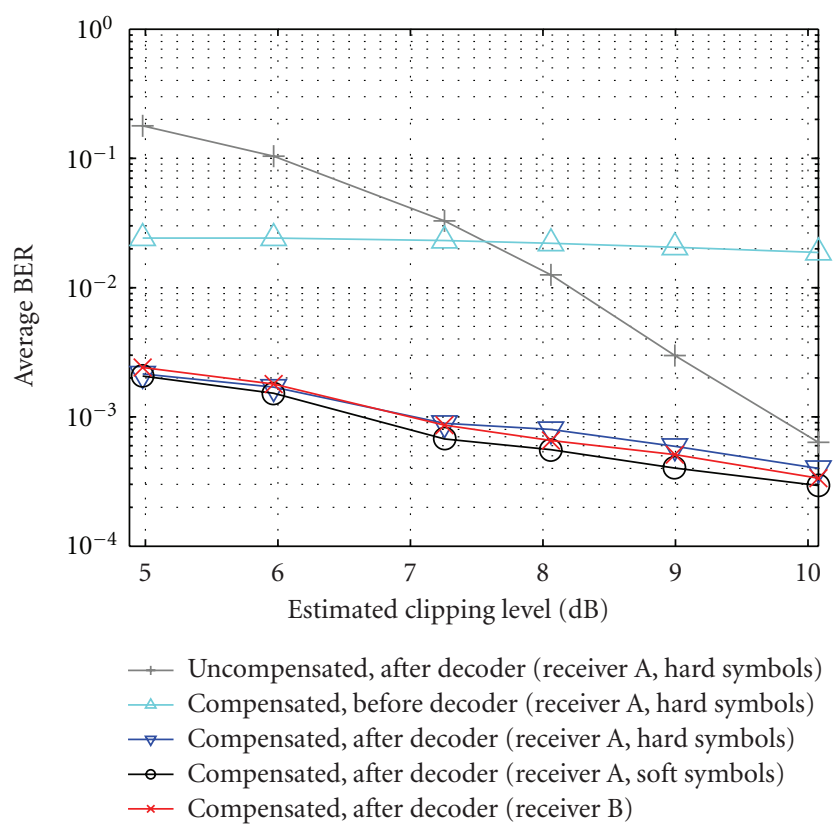

FIGURE 15: Laboratory measurement results for User 1 when $S N D R_{1}=21.7 \mathrm{~dB}$ and 5 clipping compensation iterations is performed.

between Receivers A and B is small as was indicated in the simulations of Section 4.1 .

4.3. Computational Complexity of Clipping Compensation. Preceding Sections show that, in many cases, Receiver A and $\mathrm{B}$ provide rather similar performance. However, there is a significant difference in computational complexity. This is more formally shown in Table 2 , which provides rough estimates of required real additions and multiplications per data symbol in the scenario discussed in Section 4. The numbers of operations are only suggestive since they are strongly depending on implementation platform in practice and the way how the particular functions are carried out. Only the usage of hard symbols estimates in clipping compensation is considered in this Section, because the usage of soft symbols does not change any main conclusions drawn here.

The channel estimation in Receiver A is more complex than the one of Receiver B, because it has to calculate the symbol reliabilities in (17) and combine estimates as defined in (16). For pilot-based channel estimates, the length of the used interpolation filter is 65 and $L=8$ is considered in Table 2. The complexity of the turbo decoder is defined in [28] using the so-called equivalent additions meaning that computational costs of all mathematical and logical operations are expressed as multiples of one real addition operation. The same principle is adapted to all parts of Table 2 where needed. The complexity of FFT and IFFT operations are assumed to be as defined in [29].

As can be concluded from Table 2, the most complex part of the receivers is the turbo decoder. Receiver A uses the symbol demapper and turbo decoder in every clipping compensation iteration, that is, $I$ times in total, whereas 
TABLE 2: Rough estimate for computational complexity of the clipping compensation per data symbol expressed with real addition and real multiplication operations.

\begin{tabular}{lcc}
\hline Operation & Additions & Multiplications \\
\hline Scaling with $1 / \hat{\alpha}_{1}$ & 0 & 2 \\
Channel estimation (Receiver A) & $13 M+17$ & $20+M$ \\
Channel estimation (Receiver B) & 14 & 16 \\
Channel equalization & 0 & 4 \\
Soft symbol demapping & $27 M \cdot 2^{M}$ & $3 M \cdot 2^{M}$ \\
Turbo decoding & $2130 M R$ & 0 \\
Apply channel estimate to & 0 & 4 \\
symbol estimate $\left(\hat{H}_{k} \hat{X}_{k}\right)$ & $\left(2 N \log _{2} N\right) /\left((1-1 / L) N_{A}\right)$ & $\left(N \log _{2} N\right) /\left((1-1 / L) N_{A}\right)$ \\
IFFT & $(2 N) /\left((1-1 / L) N_{A}\right)$ & 0 \\
Clipping & 0 & 2 \\
Scaling with $\hat{\alpha}_{1}$ & $\left(2 N \log _{2} N\right) /\left((1-1 / L) N_{A}\right)$ & $\left(N \log _{2} N\right) /\left((1-1 / L) N_{A}\right)$ \\
FFT & 4 & 0 \\
Calculating $\hat{Z}_{k}$ and subtracting & $(3 N-1) /\left((1-1 / L) N_{A}\right)$ & $(N+7) /\left((1-1 / L) N_{A}\right)$ \\
it from $\left.\widetilde{Y}_{k} \hat{y}_{1}\right)$ & {$\left[\left(\left(1+2 \log _{2} N\right) N+7\right) /\left((1-1 / L) N_{A}\right)\right.$} \\
Estimating $\hat{\alpha}_{1}$ & {$\left[\left(\left(5+4 \log _{2} N\right) N-1\right) /\left((1-1 / L) N_{A}\right)\right.$} & $\left.+\left(1+3 \cdot 2^{M}\right) M+32\right] I$ \\
\hline Total $($ Receiver A) & $\left.+\left(27 \cdot 2^{M}+2130 R+13\right) M+21\right] I$ & $+3 M \cdot 2^{M}$ \\
\hline Total $($ Receiver B) & {$\left[\left(\left(5+4 \log _{2} N\right) N-1\right) /\left((1-1 / L) N_{A}\right)+18\right] I$} & \\
\hline
\end{tabular}

Receiver B uses them only once. Therefore, Receiver B can be considered to be noticeably less complex. When using the parameter values from Table 1 and five clipping compensation iterations, it can be calculated from Table 2 that Receiver A requires 30865 additions and 1403 multiplications per data symbol whereas Receiver B needs only 6638 additions and 595 multiplications.

\section{Conclusions}

This paper discussed the compensation of unintentional clipping occurring in the $\mathrm{A} / \mathrm{D}$ converter of a radio receiver. A digital postprocessing compensation method iteratively exploiting the symbol decisions and clipping distortion regeneration was proposed for multiband OFDMA receivers. The performance of the method was carefully studied in fading channel circumstances using computer simulations and also verified using concrete laboratory measurements with real A/D hardware. It was shown that it is possible to recover the system performance under significant clipping almost to the level of a nonclipped situation. The performance is mostly limited by the accuracy of the channel estimation, which is compromised under heavy clipping. Although it cannot be avoided that the clipping interferes with the channel estimation, it should be studied in the future what is the optimal channel estimation scheme under heavy unintentional clipping. This paper also discussed the complexity of the proposed clipping compensation. It was proven that it is possible, without considerably decreasing the performance, to iterate the compensation process on symbol level and perform the symbol demapping and channel decoding only once after finishing the clipping compensation. This way the computational complexity of the receiver signal processing can be kept at reasonable level.

\section{Acknowledgments}

This work was supported by the Academy of Finland (under the project "Digitally-Enhanced RF for Cognitive Radio Devices"), the Finnish Funding Agency for Technology and Innovation (Tekes, under the projects "Advanced Techniques for RF Impairment Mitigation in Future Wireless Radio Systems" and "Enabling Methods for Dynamic Spectrum Access and Cognitive Radio"), Austrian Center of Competence in Mechatronics (ACCM), Jenny and Antti Wihuri Foundation, Finnish Foundation for Technology Promotion, and Tampere University of Technology Graduate School.

\section{References}

[1] J. Yang, R. W. Brodersen, and D. Tse, "Addressing the dynamic range problem in cognitive radios," in Proceedings of the IEEE International Conference on Communications (ICC '07), pp. 5183-5188, Glasgow, UK, June 2007.

[2] B. Razavi, "Cognitive radio design challenges and techniques," IEEE Journal of Solid-State Circuits, vol. 45, no. 8, Article ID 5518490, pp. 1542-1553, 2010.

[3] C. Svensson, "The blocker challenge when implementing software defined radio receiver RF frontends," Analog Integrated Circuits and Signal Processing, vol. 64, no. 2, pp. 81-89, 2010.

[4] A. Rusu, D. Rodríguez de Llera González, and M. Ismail, "Reconfigurable $\mathrm{ADCs}$ enable smart radios for $4 \mathrm{G}$ wireless 
connectivity," IEEE Circuits and Devices Magazine, vol. 22, no. 3, pp. 6-11, 2006.

[5] T. Tomioka, R. Sakata, T. Horiguchi, T. Tomizawa, and K. Inoue, "A/D converter clipping noise suppression for highsensitivity carrier-sensing of cognitive radio transceiver," in Proceedings of the 50th Annual IEEE Global Telecommunications Conference (GLOBECOM '07), pp. 4170-4174, Washington, DC, USA, November 2007.

[6] M. Allén, J. Marttila, and M. Valkama, "Modeling and mitigation of nonlinear distortion in wideband A/D converters for cognitive radio receivers," International Journal of Microwave and Wireless Technologies, vol. 2, no. 2, pp. 183-192, 2010.

[7] M. Allén, J. Marttila, and M. Valkama, "Digitally-enhanced wideband analog-digital interfaces for future cognitive radio devices," in Proceedings of the 8th IEEE International NEWCAS Conference (NEWCAS '10), pp. 361-364, Montréal, Canada, June 2010.

[8] S. H. Han and J. H. Lee, "An overview of peak-to-average power ratio reduction techniques for multicarrier transmission," IEEE Wireless Communications, vol. 12, no. 2, pp. 56-65, 2005.

[9] H. Chen and A. M. Haimovich, "Iterative estimation and cancellation of clipping noise for OFDM signals," IEEE Communications Letters, vol. 7, no. 7, pp. 305-307, 2003.

[10] H. Zhang, X.-G. Xia, Q. Zhang, and W. Zhu, "Iterative decision-aided clipping compensation and its application to scalable video transmission with multiband OFDM," IEEE Transactions on Vehicular Technology, vol. 56, no. 2, pp. 756765, 2007.

[11] D. Petrovic, W. Rave, and G. Fettweis, "Effects of phase noise on OFDM systems with and without PLL: characterization and compensation," IEEE Transactions on Communications, vol. 55, no. 8, pp. 1607-1616, 2007.

[12] V. Syrjälä and M. Valkama, "Receiver DSP for OFDM systems impaired by transmitter and receiver phase noise," in Proceedings of the IEEE International Conference on Communications (ICC'11), Kyoto, Japan, June 2011.

[13] J. Šterba, J. Gazda, M. Deumal, and D. Kocur, "Iterative algorithm for nonlinear noise cancellation and channel reestimation in nonlinearly distorted OFDM system," in Proceedings of the 8th International Symposium on Applied Machine Intelligence and Informatics (SAMI '10), pp. 65-70, Herl'any, Slovakia, January 2010.

[14] M. Allén, J. Marttila, and M. Valkama, "Iterative signal processing for mitigation of wideband ADC nonidealities in cognitive radio receiver," in Proceedings of the 19th European Signal Processing Conference (EUSIPCO '11), pp. 2279-2283, Barcelona, Spain, August 2011.

[15] J. J. Bussgang, "Crosscorrelation functions of amplitude distorted Gaussian signals," Tech. Rep. 216, MIT Research Laboratory of Electronics, Cambridge, Mass, USA, 1952.

[16] H. Ochiai and H. Imai, "Performance analysis of deliberately clipped OFDM signals," IEEE Transactions on Communications, vol. 50, no. 1, pp. 89-101, 2002.

[17] H. E. Rowe, "Memoryless nonlinearities with gaussian inputs: elementary results," The Bell System Technical Journal, vol. 61, no. 7, pp. 1519-1525, 1982.

[18] H. Ochiai and H. Imai, "Performance of the deliberate clipping with adaptive symbol selection for strictly band-limited OFDM systems," IEEE Journal on Selected Areas in Communications, vol. 18, no. 11, pp. 2270-2277, 2000.

[19] L. Cheded, "Invariance property of gaussian signals: a new interpretation, extension and applications," Circuits, Systems, and Signal Processing, vol. 16, no. 5, pp. 523-536, 1997.
[20] C. Berrou, A. Glavieux, and P. Thitimajshima, "Near Shannon limit error-correcting coding and encoding: turbo-codes," in IEEE International Conference on Communications (ICC '93), pp. 1064-1070, Geneva, Switzerland, May 1993.

[21] D. Divsalar and F. Pollara, "Turbo codes for PCS applications," in Proceedings of the IEEE International Conference on Communications (ICC '95), pp. 54-59, Seattle, Wash, USA, June 1995.

[22] 3rd Generation Partnership Project, "3GPP TS 25.212 V7.2.0, technical specification group radio access network, multiplexing and channel coding (FDD), Release 7," 2006, http://www. 3gpp.org/ftp/Specs/2006-09/Rel-7/25_series/25212-720.zip.

[23] P. Robertson, E. Villebrun, and P. Hoeher, "Comparison of optimal and sub-optimal MAP decoding algorithms operating in the log domain," in Proceedings of the IEEE International Conference on Communications (ICC '95), pp. 1009-1013, Seattle, Wash, USA, June 1995.

[24] C. L. Kei and W. H. Mow, "Improved stopping criteria for iterative decoding of short-frame multi-component turbo codes," in Proceedings of the IEEE International Conference on Communications, Circuits and Systems and West Sino Expositions (ICCCAS \& WeSino Expo '02), pp. 42-45, Chengdu, China, June 2002.

[25] T. B. Sørensen, P. E. Mogensen, and F. Frederiksen, "Extension of the ITU channel models for wideband (OFDM) systems," in Proceedings of the IEEE 62nd Vehicular Technology Conference (VTC-2005-Fall), pp. 392-396, Dallas, Tex, USA, September 2005.

[26] Rohde and Schwarz, "R\&S AFQ100A/B I/Q modulation generator-data sheet," 2008, http://www2.rohde-schwarz.com/ file_10716/AFQ100A_AFQ100B_dat_sw_en.pdf.

[27] Analog Devices Inc., “AD9248 data sheet, ver. B," 2010, http://www.analog.com/static/imported-files/data_sheets/ AD9248.pdf.

[28] P. H.-Y. Wu, "On the complexity of turbo decoding algorithms," in Proceedings of the IEEE VTS 53rd Vehicular Technology Conference (VTC-2001-Spring), pp. 1439-1443, Rhodes, Greece, May 2001.

[29] S. Winograd, Arithmetic Complexity of Computations, Society for Industrial and Applied Mathematics, Philadelphia, Pa, USA, 1980. 

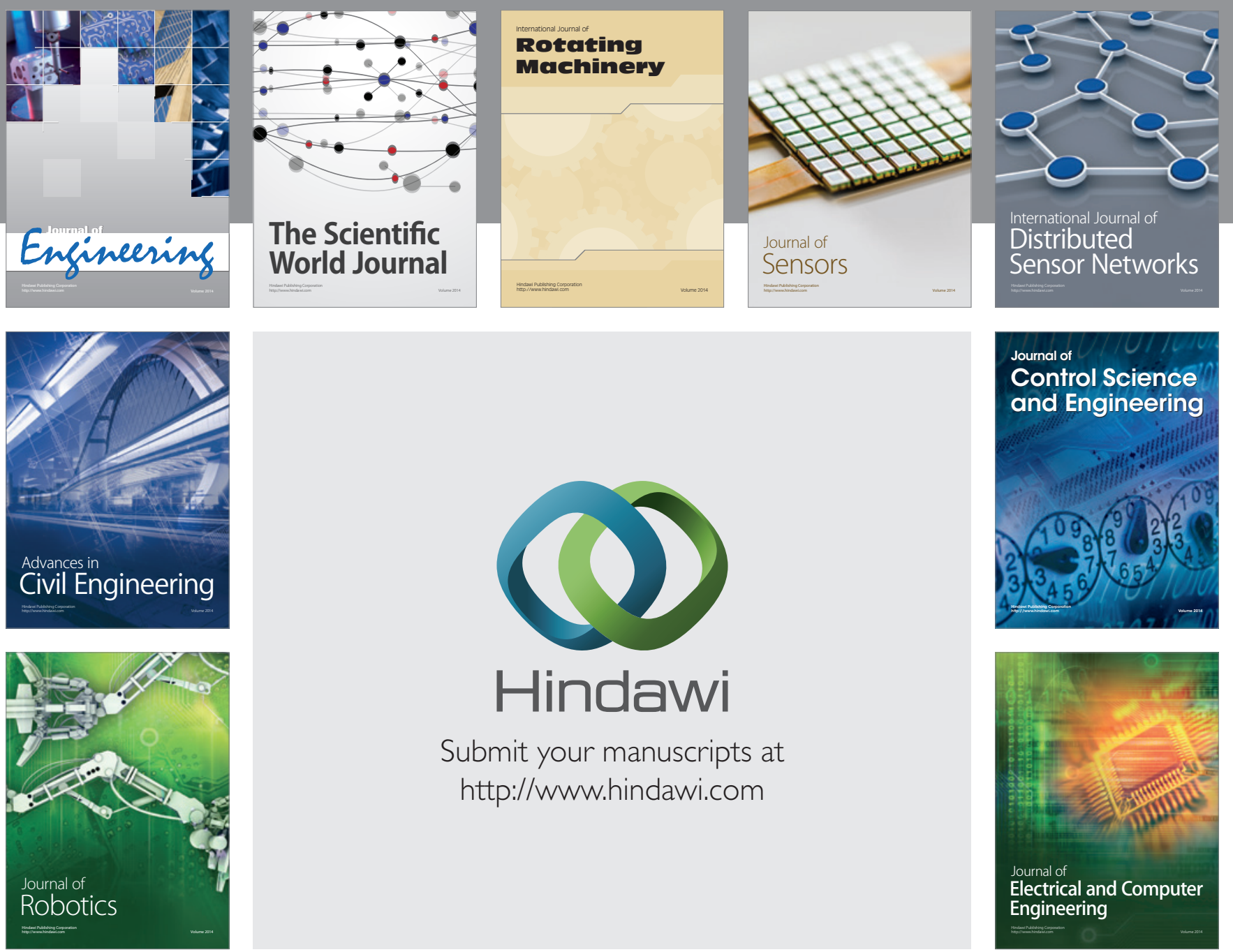

Submit your manuscripts at

http://www.hindawi.com
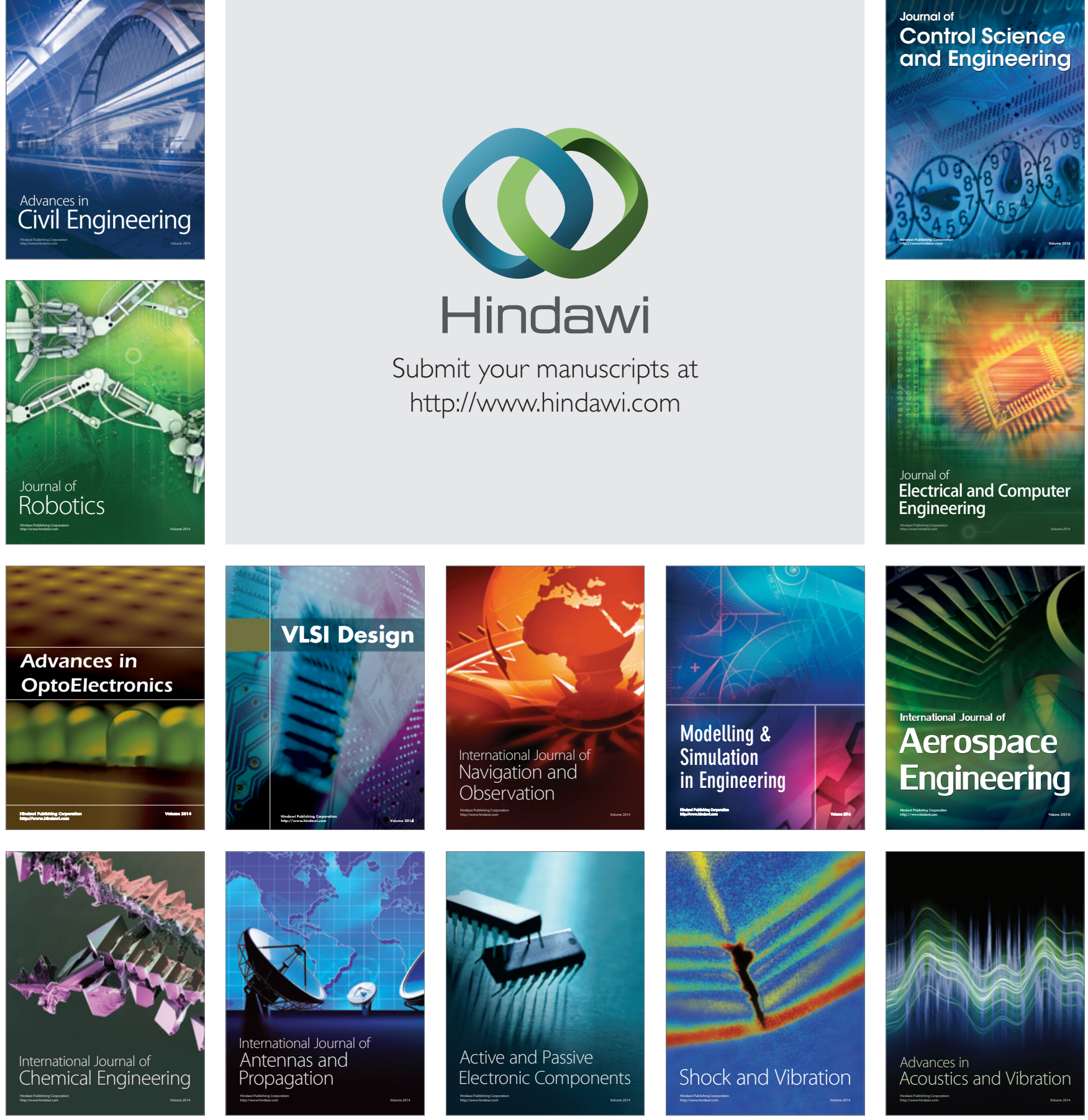